\title{
Dynamic Power Control for Wireless Backbone Mesh Networks: a Survey
}

\author{
1,2,3 Thomas Otieno Olwal, ${ }^{2}$ Barend J Van Wyk, ${ }^{1}$ Ntsibane Ntlatlapa \\ ${ }^{1}$ Meraka Institute, CSIR and ${ }^{2}$ Tshwane University of Technology \\ Box 395 Pretoria 0001 \\ Tel: +27 128412085 E-mail: thomas.olwal@gmail.com,vanwykb@gmail.com, \\ nntlatlapa@csir.co.za \\ ${ }^{3}$ Karim Djouani, ${ }^{3}$ Patrick Siarry, ${ }^{2}$ Yskandar Hamam \\ ${ }^{3}$ LiSSi, University of Paris-Est, France \\ 61 av. du Général de Gaulle, 94010 CRETEIL Cedex
}

Tél. : 01.45.17.15.67. E-mail: djouani, siarry@univ-paris12.fr, yskandar@hamam.ws

\begin{abstract}
With the tremendous growth of wireless networks into the next generation to provide better services, Wireless Mesh Networks (WMNs) have emerged to offer ubiquitous communication and seamless broadband applications. WMNs are hybrid networks composed of a mixture of static Wireless Mesh Routers (WMRs) and mobile Wireless Mesh Clients (WMCs) interconnected via wireless links to form a multi-hop wireless Ad Hoc network (WANET). WMNs are self-organized, self-configured, and reliable against single points of failures, and robust against RF interference, obstacles or power outage. This is because WMRs forming wireless backbone mesh networks (WBMNs) are built on advanced physical technologies. Such nodes perform both accessing and forwarding functionality. They are expected to carry huge volumes of traffic and be "on power" at all times. While trying to increase network capacity, problems of the dynamic transmission power control (DTPC) arise in such networks. Such problems include RF Interference, Connectivity and energy-depletion. While there are numerous studies on this topic, contributions in the context of WBMNs are still challenging but interesting research areas. This paper provides an overview of the DTPC algorithms central to the WBMNs framework. The open issues are also highlighted.
\end{abstract}

Keywords: Dynamic Power Control (DTPC) Algorithms, Link-Layer Protocol, Wireless Backbone Mesh Networks (WBMNs), Wireless Mesh Networks (WMNs). 


\section{Macrothink}

\section{Introduction}

With the tremendous growth of wireless networks into the next generation to provide better services, an important technology, wireless mesh networks (WMNs), has emerged recently. WMNs have gained immense research interests from the wireless networks' research society in the past decade. This sudden interest emanates from developments which indicate that the WMNs can offer ubiquitous communication and seamless broadband applications. Architecturally, WMNs are hybrid networks composed of a mixture of static and mobile nodes interconnected via wireless links to form a multi-hop wireless ad hoc network (WANET). The static nodes are usually called wireless mesh routers (WMRs) while mobile nodes are usually called wireless mesh clients (WMCs) as shown in Fig. 1. While static WMRs form the wireless backbone mesh networks (WBMNs), the WMCs access the Internet through WBMNs as well as directly meshing with each other [1]. The WMCs form traditional mobile wireless Ad Hoc networks (MANETs) [2]. On the other hand, the high performing WMRs access the MANETs and voluntarily relay backbone traffic to the Internet via a few fixed gateways. This role makes WMNs quite distinct from the traditional wireless networks, such as the wireless sensor networks (WSNs) [3], MANETs [2], Wireless Fidelity (Wi-Fi) and cellular networks.

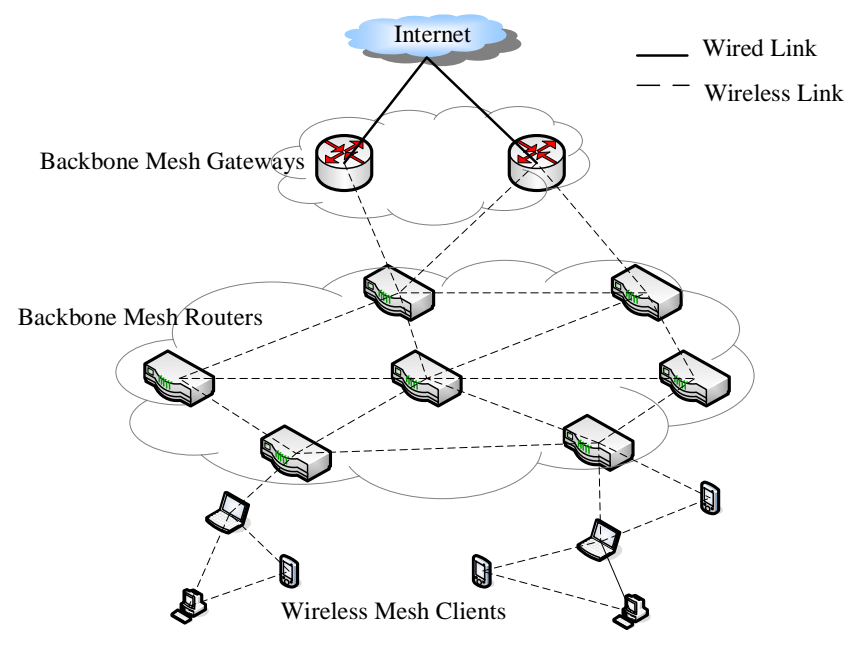

Figure 1: General wireless mesh network architecture

Numerous significant attributes of the WBMN can be outlined. First, the mesh routers (mesh points) are dynamically self-organized and self-configured. That is, the nodes in the mesh network automatically establish and maintain stable network connectivity. This feature provides the end-users with many advantages, such as low up-front cost, easy network maintenance, robustness, and reliable service coverage. Second, the WMRs provide redundant paths between the sender and the receiver of the wireless connection. This feature provides path reliability against single points of failures. It also creates the network robustness against node or path failures due to RF interference, obstacles or power outage by enhancing multiple alternative routes. Third, the WMRs are built from advanced radio 
technologies, e.g., multiple radio interfaces, software defined radios and smart antennas. These radio technologies may be built on either the same or different wireless access technologies (i.e., homogeneity or heterogeneity). The motivation is to increase the network capacity significantly. The network capacity is the sum of the throughput provided to all end-users in the network. Finally, mesh routers have gateways and bridge functionalities. This is to enable the integration of WMNs with various existing wireless network technologies, such as WSNs, Wi-Fi, WiMAX, Wireless Region Area Networks (WRANs) and cellular networks [4].

In view of these significant attributes, WMN technology has promised several emerging and commercially interesting applications. Examples include broadband home networking, community and neighbourhood networks, enterprise networking, coordinated and collaborative network management and intelligent transportation system. Indeed, WMN has now made it possible for cash strapped Internet Service Providers (ISPs) and others to roll out robust and reliable wireless broadband services at a reasonable cost [5]. Such enormous applications have triggered several challenges, though, that researchers must address before WMNs can realize its full application potential. Based on the capability to access and forward huge volumes of traffic over a long time, it is crucial to address network capacity, connectivity and energy-efficiency issues. These are the problems of the scalable Dynamic Transmission Power Control (DTPC) for the WBMNs. This is the problem of adjusting the transmission power level at each wireless radio interface on a per packet basis, based on user and network applications [6].

The DTPC problems in wireless networks have been extensively studied in literature. The ambition is not to provide a complete coverage of the area in this paper, but rather to outline some important and central contributions to the WBMNs. Some examples of the DTPC problems in distributed wireless cellular networks (WCNs) have been provided by [7-10], in wireless senor networks (WSNs) by [3, 11-13] and in wireless Ad Hoc networks (MANETs) by [14-17]. In the recent past, the joint DTPC and network cross-layer resource optimizations have been addressed for wireless mesh networks (WMNs) [18-20]. In general, the DTPC problem can be assumed to be an optimization formulation irrespective of the solution techniques considered [7]. Thus, the power control optimization is purely described by four tuples: optimization variables, objective function, constraint set, and constant parameters.

The remainder of the paper is organized as follows. In section 2, taxonomy of dynamic power control (DTPC) algorithms is presented. Section 3 addresses Mathematical Programming based DTPC Algorithms. In section 4, the DTPC Algorithms based on Game-Theory are provided. Section 5 discusses Dynamic Control Theoretical Algorithms. In section 6, Algorithms based on Heuristics at layers of protocol stack are presented. Section 7 concludes the paper and gives open issues for further study.

\section{Taxonomy of Dynamic Power Control Algorithms}

As a consequence of the optimization formulations, this section presents an overview of the proposed approaches or algorithms to the DTPC problems for WBMNs and classifies them according to the solution techniques. The broad classification includes: Mathematical 
programming theory, Game-theory, Dynamic control theory and Network Protocol Heuristics. Some past contributions in these categories are: Mathematical programming (e.g., [6, 21-23]), Branch and Bound [24, 25], Protocol Heuristics (e.g., [15, 17, 26-32]), Simulated annealing [33], Game theory (e.g., [34-37]), predictive methods (e.g., [6, 10, 38]), Kalman Filter methods (e.g., [39-41]) and Cross-layer approaches (e.g., [21, 42]). These techniques for the WBMNs may be classified hierarchically as shown in Fig. 2.

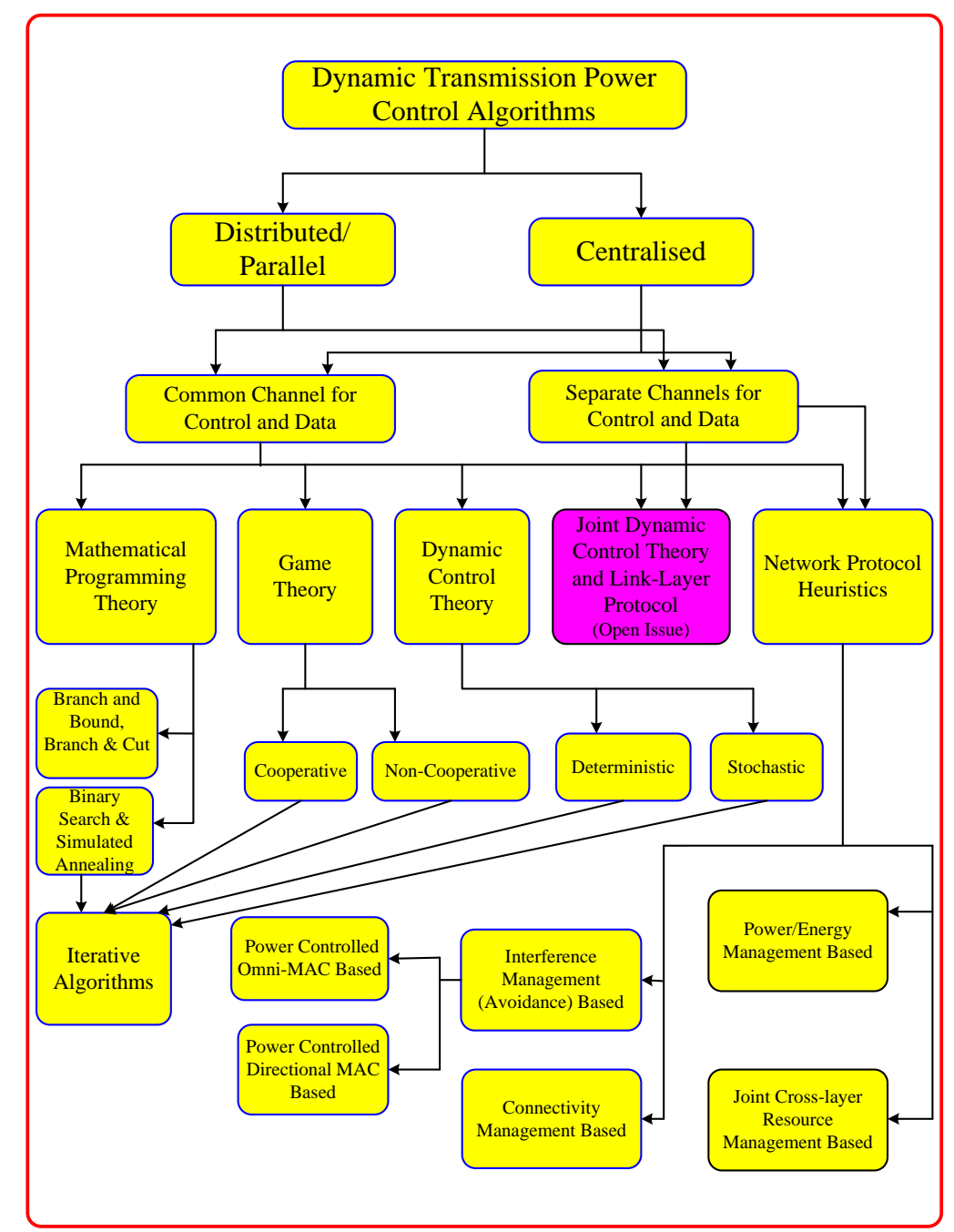

Figure 2: Taxonomy of DTPC Algorithms

\section{Algorithms based on Mathematical Programming Techniques}

In wireless cellular or Ad Hoc networks, the quality of service (QoS) is interference-limited [22]. Therefore most of the DTPC problems can be formulated as optimization problem composed of a cost function subject to a set of constraints imposed both by the service or application and the network or system. For example, the DTPC problem can be formulated as a nonlinear optimization with a system-wide objective, e.g., maximizing the total system throughput or the worst user throughput, subject to QoS constraints from individual users, e.g., on data rate, delay, and outage probability [22]. 
However, in a multi-hop wireless system, the optimal DTPC problem is known to be an NP-hard and the time complexity of the optimal algorithm increases rapidly with the problem size, i.e., the number of power levels and nodes in the system. Due to complexity of the DTPC problem, most of the work in the literature developed heuristics while optimal algorithms are developed only for restricted cases or for small problems. There have been many efforts in solving Signal-to-Interference plus noise ratio (SIR or SINR) based power optimization problems. Some algorithms minimize total transmission power consumption over a set of discrete available power levels (e.g., [43]). Still other algorithms seek to minimize outage probability of the SIR (e.g., [24, 44]).

In [45], Tang et al., examined joint link scheduling and power control with the objective of throughput maximization. The maximum throughput link scheduling with power control has been formulated as a mixed integer linear programming (MILP) problem so as to provide optimal solutions. To obtain a good trade-off between throughput and fairness, a new parameter called the demand satisfaction factor (DSF) has been defined and an effective polynomial-time heuristic algorithm developed. Numerical results showed that the bandwidth can be fairly allocated among all links or flows by solving the MILP formulation or by using the heuristic algorithm. The drawback of this model is that centralised arbiters that allocate bandwidth are assumed and thus power expense needs to be accounted for. The MILP formulations have also been used by Ramamurthi et al., [46], to study link scheduling and power control in WMNs with directional antennas. Although the use of directional antennas allows network fairness in bandwidth usage, the heuristic algorithms derived perform optimally only at light traffic loads. This implies that, there can be no performance guarantee at heavy traffic loads.

Based on broadcasting in wireless networks so as to reach several nodes with a single transmission, Montemanni et al., [23, 33, 47, 48] presented a minimum power symmetric connectivity problem in wireless networks. The minimum power broadcast (MPB) problem requires the assignment of transmission powers to the nodes in such a way that the network is connected and the total power consumption is minimized. To achieve this goal, authors have formulated the problem as a mixed integer programming (MIP) and applied different solution heuristics. In [47], the branch and cut algorithms have been used. In [33], Montemanni et al., have adopted a simulated annealing to obtain sub-optimal solutions. In [48], the authors presented a comprehensive review on different solution techniques to the MPB problem in wireless networks. Montemanni performed several comparative simulation tests against the broadcast incremental power (BIP) algorithms [49]. The BIP was based on constructing minimum power broadcast trees by adding new nodes to the tree on a minimum incremental cost basis until all intended destination nodes are included. The simulation results showed that MPB outperformed the BIP algorithms in terms of energy-efficiency. It should be noted that, broadcasts results in message duplications and thus network flooding problems.

Numerous studies on Integer Linear Programming (ILP) for power optimization formulations have been conducted by (e.g., [7, 18, 24, 32, 44, 46]). Power and rate control outage based under multiple access interference (MAI) and heterogeneous traffic sources have been proposed by [24, 44]. The authors formulated the total rate maximization problem by a base receiver station for all nodes. They set the SINR outage probability, total 
transmission power and achievable rate as the model constraints. In order to solve this problem, authors have adopted two power control algorithms. The outer and inner loop power control algorithms. The outer loop power control is responsible for setting the target SINR at the receiver input. The inner loop control compensates channel variations induced by fast fading phenomena. The inner loop tries to adjust the powers of each node in order to meet a target level for the SINR. The optimal solution has been obtained using the branch and bound (BB) criteria.

From a different perspective, Tadonki and Jose Rolim [32, 50] have solved the dual power management problem in wireless sensor networks (WSN). The authors have considered a given network scenario with two possible transmission powers (e.g., low and high). They have investigated the problem of finding a minimum size subset of nodes such that if they are assigned high transmission power while the others are assigned low transmission power, then the network will be strongly connected. Simulation results of the exact solutions yielded efficient execution time and solution quality. The approach has assumed, however, that each node has only two powers and thus a restrictive assumption. In order to formulate scheduling problems using the ILP techniques, Capone and Carello [51] have addressed both power control and rate adaptation. The authors set an objective function of minimizing the number of used time slots subject to transmission power constraints as an ILP. They used a column generation approach to solve the minimal power variables. Column generation that computes a lower bound for the problem and the heuristics that compute feasible integer solutions were suggested. Numerical results have shown that the column generation approach can solve small size instances while for bigger problems solving the pricing problem to optimality becomes difficult and therefore the whole approach becomes slow. This method has assumed a centralized arbiter to maintain and distribute time slots across the system and thus overhead costs that exacerbate retransmission problems [18].

In order to achieve power optimization in low SINR channels, Vasudevan et al., [52] have formulated an optimization problem to optimally assign power to nodes in the data-gathering tree for maximizing the lifetime of the tree, which is equivalent to the time until network partition due to battery outage. In order to solve this problem, they have proposed a Binary search algorithm for optimal power assignment among nodes that maximizes the tree lifetime. Using turbo codes as an example of a channel coding technique that compensates for a low SINR channels, they have demonstrated significant improvements in network lifetimes. However, turbo codes are computationally complex techniques and aspect of time costs in running binary search has not been investigated either.

For a generalized SIR regime, Chiang [21] have presented a distributed power control algorithm that couples with existing transmission control protocols (TCPs) to increase end-to-end throughput and energy efficiency of the network. Authors have first formulated a nonlinear constrained utility maximization with "elastic" link capacities. They have then proved the convergence of the coupled algorithm to the global optimum of the joint power control and congestion control, for both synchronised and asynchronous implementations. They have found out that the rate of convergence is geometric and a desirable modularity between the transport and physical layers can be maintained. Both analytic and simulation results have illustrated the robustness of the algorithm to channel-outage and to path-loss 
estimation errors. However, the algorithm trades-off implementation simplicity for performance optimality. The authors in [22] have shown that in the high SIR regime, these nonlinear and apparently difficult, non-convex optimization problems can be transformed into convex optimization problems in the form of geometric programming (GP). In the medium to low SIR regime, some of these constrained nonlinear optimizations of power control cannot be turned into tractable convex formulations, but a heuristic can be used to compute in the most cases the optimal solution by solving a series of GPs through the approach of successive convex approximations. These techniques for power control, together with their implications to admission control and pricing in wireless networks, are illustrated through several numerical examples. However, GP method is effective to certain applications but requires high SIR channels [20]. The use of a convex GP for which Interior Point Method algorithm can be applied is investigated by [53]. The authors have generalized the problem of joint optimization of transmit power-time and bit energy efficiency using convex optimization. The nonlinearity of the problem makes it difficult to solve at reasonable low energy levels.

\subsection{Summary}

In summary, mathematical programming theory (MPT) provides a generalized tool for formulating the DTPC problem. However, feasible optimal solutions may not exist depending on the dimension of the problem. Such situations require sub-optimal heuristics. Furthermore, the MPT may not yield reliable solutions in stochastic wireless channels, unless robust filters are implimented. Based on its generalization attribute, the MPT can be invoked to formulate the DTPC problems of the WBMNs and related algorithms may be developed to solve these problems.

\section{Algorithms based on Game-Theoretical Techniques}

In wireless communication systems, the transmission power of each user (or link) contributes to the interference seen by the other users [54]. Given that there is a limited battery power available at each node but each user demands unlimited utility satisfaction, effective and efficient power control strategies ought to be in place [55]. These strategies may be designed to achieve user oriented quality of service (QoS) or system capacity (network oriented) objectives or both [56]. In order to address the strategic DTPC problems, Srivastava et al., [35] assessed game-theoretic analysis of Ad Hoc networks in terms of power control and waveform adaptation, medium access control (MAC), routing decisions, and node participations. Game theory is the ability to model individual, independent decision makers (IDMs) whose actions potentially affect all other IDMs in the system. Game-theoretic models analyze existing protocols and resource management schemes, and design equilibrium-inducing mechanisms that provide incentives for individual IDMs to behave in socially-constructive ways. Game theory is broadly categorized as cooperative game and non-cooperative game [43]. Cooperative game is a competition between coalitions of IDMs while non-cooperative game is a competition between individual IDMs. Numerous DTPC 
publications based on the cooperative game can be found in (e.g., [43, 57-60]) and those for non-cooperative game are found in (e.g., [61]). According to the assessment by Koskie [55], most of past contributions have contextualized game theory for mobile nodes. We outline the DTPC based on game theory in the context of WBMNs.

\subsection{Cooperative Game Based Algorithms}

Goodman and Mandayan [57] proposed a utility-based network assisted power control (NAPC). The power control algorithm was implemented through SIR balancing with the assistance of the network that broadcasts the common SIR target for all users. This means that NAPC requires coordination by the network, which has to inform terminals of the best target SIR for current conditions. The utility levels achieved with NAPC are comparable to those achieved with a non-cooperative power control game with pricing [62]. However, network broadcasts cause network flooding problems [29]. The main drawback is that NAPC requires coordination by the network through broadcasts and thus NAPC lacks fault-resilience.

Jean and Jabbari [60] presented a game-theoretic delay-sensitive multi-rate power control for CDMA wireless networks with variable path-loss. In order to arrive at this goal, authors have applied stochastic game-theory which models the dynamism of the cellular uplink power-control problem. A vector of buffer states are assigned to the nodes and it captures the statistics of packet arrivals from higher layers and packet departures via successful transmissions, neither of which is deterministic. They then evaluate stationary Nash equilibrium that is a function of buffer level variation in time (i.e., congestion) and that allow for multi-rate transmission in evolving channel conditions. In both static path-loss and dynamic shadowing path-loss environments, the stochastic game equilibrium calculated compares very favourably to traditional single-agent and often approaches the performance of centralised optimization. The assignment of buffer states and CDMA codes across the system requires centralized base stations and thus signalling overhead costs [7].

In [58], authors have studied the performance of a distributed and asynchronous power control scheme for a spread spectrum wireless Ad Hoc network. In this scenario, network users exchange prices that reflect their loss in utility due to perceived interference. The prices are then used to determine optimal (i.e., utility maximizing) power levels for each user. Simulation results have shown that with logarithmic utilities, the pricing algorithm exhibits rapid convergence to the unique optimal power allocation. In another contribution, Huang et al., [58] have considered a distributed power control scheme in a spread spectrum (SS) wireless ad hoc network, in which each user announces a price that reflects his current interference level. Authors have assumed that node users voluntarily cooperate with each other by exchanging interference information. Given these prices, the authors presented an asynchronous distributed algorithm for updating power levels, and provided conditions under which this algorithm converges to an optimal power allocation. This algorithm was then linked to myopic best response updates of a fictitious game. Its convergence was characterized using super-modular game theory. Super-modular games are those characterized by "strategic complementarities". This means that when one IDM takes a 
higher action, the others want to do the same. However, motivations behind voluntary cooperation among nodes have not been properly substantiated in this work.

Altman et al., [34] have considered the situation where $N$ nodes share a common access point. With each node there is an associated buffer and channel state that change in time. Each node dynamically chooses both the power and the admission control to be adopted so as to maximize the expected throughput, which depends on the actions and states of all the nodes, given its power and delay constraints. Using Markov Decision Processes they analysed the single node optimal policies under different model parameters. In [43], an uplink power control problem where each node wishes to maximize its throughput (which depends on the transmission powers of all nodes) but has a constraint on the average power consumption has been considered. Authors assumed that a finite number of power levels are available to each wireless node. Also, the decision of a node to select a particular power level depends on its channel state. Two cases are considered: the case of full state information and the case of local state information. In each case, the authors have proposed both cooperative and non-cooperative power control. However, cellular radio system has been assumed even in the case of local state information and thus the method lacks scalable attributes of multi-hop WBMNs.

\subsection{Non Cooperative Game Based Algorithms}

Koskie and Zapf [56] have shown how the power control problem in wireless networks is formulated as a non-cooperative game in which users choose to trade off between SIR error and transmission power usage. That is, minimizing the SIR error at the cost of high transmission power usage. They have presented distributed power control strategies based on the use of Newton iterations having third-order rather than quadratic convergence. A realistic CDMA cell model has been used to simulate the proposed algorithms. Simulation results indicated that the use of Newton iterations to accelerate the convergence of the static Nash power control algorithm significantly decreased the number of iterations required for convergence. The advantage of the third-order algorithms over the second order algorithms appeared to eliminate the slight overshoot observed in early iterations. It should be noted that CDMA cell model requires centralized arbiter and thus procedure on how independent users were assigned codes and with what power level was not discussed by the authors.

In [61], a game-theoretic approach to energy-efficient power control in multicarrier CDMA systems has been proposed. In multi-carrier direct-sequence CDMA (DS-CDMA), the data stream for each user is divided into a multiple parallel streams. Each stream is first spread using a spreading sequence and then transmitted on a carrier. Therefore multi-carrier CDMA combines the benefits of orthogonal frequency-division multiplexing (OFDM) with those of CDMA for the next-generation high data-rate wireless systems. In order to achieve their goal, Meshkati et al., [61] have formulated power control problem as a non-cooperative game in which each user decides how much power to transmit over each carrier to maximize its own utility. The utility function considered measures the number of reliable bits transmitted over all the carriers per joule of energy consumed. The utility function reflects the user's preference regarding the SIR and the transmitter power [37]. It was shown by 
Meshkati et al., [61] that for all linear receivers including the matched filter, the de-correlator, and the minimum-mean-square-error detector, a user's utility is maximized when the user transmits only on its "best" carrier. This is the carrier that requires the least amount of power to achieve a particular target signal-to-interference-plus-noise ratio (SINR) at the output of the receiver. Conditions have been given that must be satisfied by the channel gains for a Nash equilibrium to exist. The distribution of users among the carriers at equilibrium was also characterised. An iterative and distributed algorithm for reaching the equilibrium (when exists) was presented. It has been shown that the proposed approach results in significant improvements in the total utility achieved at equilibrium compared with a single-carrier system and also to a multicarrier system in which each user maximizes its utility over each carrier independently. However, the proposed technique trades-off complexity for optimality and thus efficient power consumption is hard to guarantee.

In [63], a game-theoretic power management in multi-input multi-output (MIMO) Ad Hoc networks has been proposed. The power allocation at each user is built into a non-cooperative game where a utility function is identified and maximized. Due to poor channel conditions, some users have very low data transmission rates even though their transmit powers are high. Therefore, a mechanism for shutting down link users is proposed in order to reduce co-channel interference and improve energy-efficiency. Compared to multiuser water-filling and gradient projection methods (e.g., [61]), the proposed game-theoretic approach with the link user shut-down mechanism allows the MIMO ad hoc network to achieve the highest energy and the highest system capacity.

Xing and Chandramouli [37] have proposed a stochastic learning solution for distributed discrete power control game in wireless data networks. The authors have first noted that a simple discretization of the continuous transmitter power level does not guarantee convergence and uniqueness. Consequently, they have proposed two probabilistic power adaptation algorithms and analysed their theoretical properties along with the numerical behaviour. The distributed, discrete power control problem has been formulated as an N-node, non-zero sum game by [35]. In this game each user evaluates a power strategy by computing a utility value. This evaluation is performed using a stochastic iterative procedure. Authors [37] have approximated the discrete power control iterations by an equivalent ordinary differential equation and proved that the proposed stochastic learning power control algorithm converges to a stable Nash equilibrium [35]. The drawback is that the convergence times may be too long relative to the packet duration.

Thomas et al., [64] have presented a cognitive network approach to achieve the objectives of power and spectrum management. The authors cast the problem as a two phased non-cooperative game and used the properties of potential game theory to ensure the existence of, and convergence to, a desirable Nash Equilibrium. Although this is a multi-objective optimization and the spectrum problem is NP-hard, this selfish cognitive network constructs a topology that minimizes the maximum transmission power while simultaneously using, on average, less than $12 \%$ extra spectrum, as compared to the ideal solution. With a related formulation, Closas et al., [65] use a non-cooperative game theory to design a fully distributed network topology control algorithm using optimal transmit adjustment. Simulation results have shown that for a relatively low node density, the 
probability that the proposed algorithm leads to a connected network is close to one.

Huang et al., [66] have proposed two auction mechanisms, the SINR auction and the power auction, that determine relay selection and relay power allocation in a distributed fashion. For a single relay network case, the authors show that the power auction achieves the efficient allocation by maximizing the total rate increase and the SINR auction is flexible in trading off fairness and efficiency. For both auctions, the distributed best response bid updates globally converged to the unique Nash Equilibrium in a completely asynchronous manner. Same results were obtained considering generalised networks with multiple relays. Using similar approach, Jindal et al., [67] considered non-iterative power control algorithms with mutually interfering users and a common target SINR. Each transmitter knows the channel quality of its intended receiver, but has no knowledge of (potential) interference from other transmitters. The authors have considered fractional power control policies that fall between channel inversion (i.e., full channel compensation, 1/H) and constant transmission power (i.e., no channel compensation, $\mathrm{H}=1$ ). They have considered a spatially distributed (decentralized) network, representing either a wireless Ad Hoc network or unlicensed spectrum usage by many nodes ((e.g., Wi-Fi or spectrum sharing systems)). Disadvantages of iterative power controls are, for example, a feedback channel with the required latency may not be available or convergence times may be too long relative to packet duration. However, it can be argued that choosing only the fractional or constant transmission power levels are restrictive.

\subsection{Summary}

In summary, non-cooperative games are quite useful in formulating decentralized DTPC problems but finding the Nash Equilibrium in an error-prone wireless system is NP-hard [34]. Though cooperative games have been proposed, voluntary actions taken by an IDM in order to favour others, is difficult to motivate in real life [43]. Even the Nash Bargaining Solution (NBS) that achieves a good compromise between fairness and efficiency with a small system dimension guarantees no better performance with an expanded system dimension. Based on the expanded dimension of the link state information (LSI) affecting optimality of the DTPC, the required feedback latency may not be available at each IDM [8]. It is crucial to address a coalition and individual scope objective function formulation having the benefits of the two types of games. The use of dynamic control techniques to solve the problem formulations are interesting open issues.

\section{Algorithms based on Dynamic Control Theory}

The DTPC is an important means to reduce mutual interference between the users (i.e., by lowering power), while compensating for time-varying propagation conditions (i.e., by raising power) [68]. The transmission powers are controlled using feedback and feedback result in a dynamical behaviour that critically affects the network performance (i.e., throughput and delay) [69]. Methods from control theory have been used to analyse the dynamical effects and to design appropriate control strategies (e.g., [8]). The basic block 
diagram for the DTPC problem formulation with interpreted signals is illustrated by Fig. 3 . The DTPC algorithm updates transmission power levels by using quality related measurements from the environment. The output from wireless radio system (plant) is exacerbated by time-varying external disturbances such as channel states and interference. The DTPC algorithms based on such time-varying random channels for wireless cellular systems have been researched extensively (e.g., [7, 8, 68-70]). The objectives are to achieve desired quality measures [71].

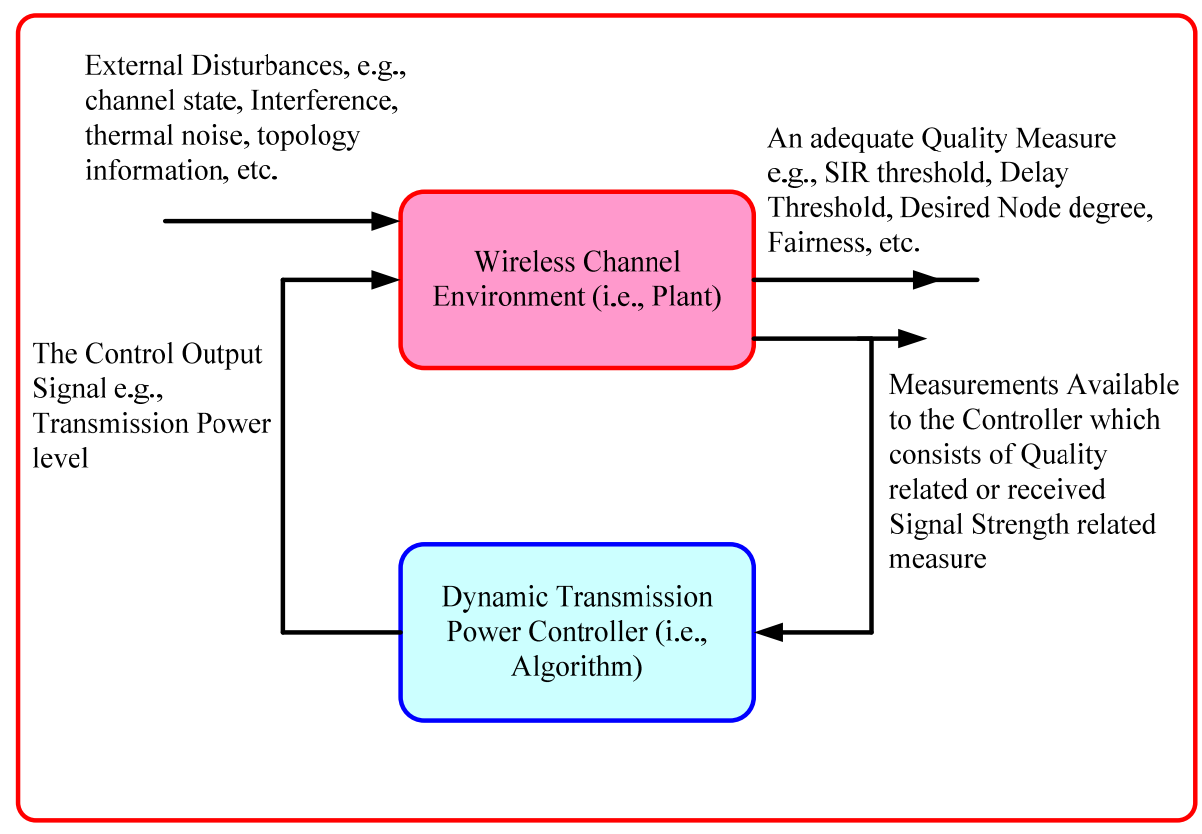

Figure 3: The Basic Block Diagram (Adapted from [8])

In [8], Gunnarsson has motivated the need to compute powers locally for each wireless connection. Methods from control theory have been used to analyse existing algorithms locally and to design local controllers with improved performance. On a global level, this author has provided results on stability and convergence of the designed controllers. The results have been illustrated by simulations using both small and large-scale simulation environments. However, the algorithms are mainly based on only the SIR measurements by the base station from the connected node. The approach limits its application to single-hop wireless networks whereby hidden terminal problems (HTPs) are not dominant. In [6] both user-centric and network-centric power control objectives have been considered. Each user adjusts the transmission power level dynamically based on the network interference and SINR measurements. Both the interference and SINR are predicted before adjusting the transmission power to an optimal level. The robustness of algorithm has been investigated against time-varying and noisy channel conditions. The advantage of adapting the transmission power in this way is that, a user can choose either to be greedy or energy efficient. Greedy users tend to increase their transmission power levels so as to maximize their SINR. On the other hand energy-efficient users tend to lower or possibly power down so as to save their transmission power. The method, however, relies on the base station to assign individual users CDMA codes. The aggregate interference is estimated due to forward 
transmissions. In contrast, WBMNs would require bidirectional control signalling whereby aggregate interference takes care of the backward transmission as well.

In [72] provided for a distributed on-line power control of Ad-Hoc networks with user-specific SIR requirements. Their algorithm yields the minimum transmitter powers that satisfy the SIR requirements. The only draw back is that their algorithm requires that the channel gains between nodes in the Ad Hoc networks be constant during the transmission power adaptation. This is reasonable only in case the time scale for transmission power adaptation is much faster than the time scale of the channel variability (i.e., stationary users, slowly-varying channels). Yates [73] extended on the work by Foschini and Miljanic, [72], whereby each user is iteratively assigned to a fixed base station at which it's SIR is highest. Authors have developed a general proof of the synchronous and totally asynchronous convergence of predicted power iteration. However, it can be inferred that a centralized receiver base station assigns orthogonal codes to nodes. The centralized controller has knowledge of the channel states for all users in the system [70]. Nonetheless, such a method is too restrictive to apply to decentralized WBMNs.

Holliday et al., [70] discussed the power control problem and its relation to the call admission problem for Ad Hoc networks. They have pointed out that the power control design in wireless networks depends on channel condition dynamics. As a result they have developed an appropriate stochastic approximation power optimality algorithm. This algorithm is modified to track non-stationary equilibrium (i.e., users entering and leaving the system) that performs an admission control. They have also suggested that the iterations of the stochastic approximation algorithms can be decoupled to form fully distributed online power and admission control algorithms for ad hoc wireless networks with time-varying channels. However, the algorithm has been based on the physical layer channel conditions. Algorithms that resolve both rate and power control problems are suggested by Subramanian and Sayed [42]. Formulations are based on state space model with and without channel condition uncertainties [74]. The algorithms have been found robust against SIR measurement uncertainties for single hop channels [69].

In $[69,75]$, the stochastic dynamic optimization technique has been used to minimize the total combined mobile receiver operating in log-normal fading channels. A similar approach was followed by [76] to adaptively optimize the quantization of the feedback SIR. In the same context, Neely et al., [68] exploited the convex optimization theory to study the dynamic power allocation and routing for time-varying wireless networks. Their main contributions were the formulation of a general power control for time-varying wireless networks, the characterization of the network layer capacity region and the development of capacity achieving routing and power allocation algorithms. These algorithms hold for systems with general arrival and channel processes and offer delay guarantees at the queuing systems. However, these algorithms required centralised arbiter and thus not feasible for Ad Hoc networks [77].

Based on the convex formulations, Olama et al., [69] have suggested stochastic power control model for time-varying long term fading (TV LTF) wireless networks. The TV LTF captures both space and time variations. The proposed TV LTF is represented by stochastic differential equation driven by Brownian motion. The Brownian motion allows viewing the 
wireless channel as a dynamical system solvable by adaptive and non-adaptive estimation techniques. Using similar formulations, Hande et al., [78] have solved the joint power control and SIR assignment problem. Authors resolve the coupled constraint set problem by using a re-parameterization approach and the left Perron Frobenius eigen-vectors. They have developed a distributed algorithm that can achieve any Pareto-optimal SIR assignment, then a distributed algorithm that picks out a particular Pareto-optimal SIR assignment and the associated powers through utility maximization [7].

Adaptive predictive power control for the uplink channel in DS-CDMA systems has been proposed by [79]. The authors have first analysed the conventional closed-loop power control system. The analysis has been performed using a prediction technique for estimating the channel-power fading profile. This prediction technique is based on oversampling the power measurements. The proposed scheme performs significantly in terms of minimizing the power-error variance. The main draw back with Adajani and Sayed [79] predictive algorithm is that base stations are required in order to provide the nodes with future channel states based on the previous and present measurements. This power control algorithm does not scale properly as the network density in a geographical cell increases [77]. Shoarinejad et al., [80] have proposed integrated distributed predictive power control and dynamic channel assignment (DCA). This has been achieved by first deriving a minimum interference DCA algorithm. They then design Kalman filters in order provide the predicted measurements of both the channel gains and the interference levels. The predicted information is in turn used to update the power levels. Local and global stability of the network have been analysed and extensive computer simulations carried out to show the improvement in performance, under the dynamics of user arrivals and departures, and base station handoffs. It has been observed that call droppings and call blockings are decreased while, on average, fewer channel reassignments per call are required. The main drawback is that the approach has been investigated under the centralised FDMA and TDMA schemes. Transmission power levels for distributing such control signalling in real-time has not been outlined [38, 81].

Leung [39-41, 82] proposed transmission power control algorithms based on tracking of the interference power at the receiver by a Kalman filter. In [82] link adaptation and power control for streaming services was investigated by Leung et al. In [41], Leung remarked that power control algorithms proposed earlier for packet-switched time division multiple access (TDMA) wireless networks, do not yield performance gain for short message length and/or moderate control delay [39]. As a consequence, they have introduced an error margin in determining the transmission power obtained from tracking the interference prediction error [41]. This algorithm has been later applied to packet voice service applications [83]. It has been found out that by introducing such error margin, SINR performance can significantly improve even for short message (i.e., yields little temporal correlation for the interference prediction) and for control delay (i.e., incurred in measuring the interference power and passing the power control information from the receiver to the transmitter). The medium access control allows at most one terminal in each sector or cell to send data at a time. Furthermore, the base station knows which terminal is scheduled to transmit at different times. In general wireless Ad Hoc networks, random medium contentions are dominant and there is a need to have power control taking into account such phenomena. 
Koskie and Gajic [9] have generalized the main results on SIR based power control algorithms in wireless radio systems. Their aim was to increase network capacity, extend battery life, and improve quality of service (QoS). Recent approaches solving power distribution problem have exploited Kalman filters, dynamic estimators, and non-cooperative Nash game theory. In this context, Koskie and Gajic [10] have presented optimal SIR-Based power control strategies for wireless CDMA networks. They have designed control algorithms that explicitly consider the trade-off between the cost of transmit power and SIR error. In this case the cost of each node consists of a weighted sum of power, power update, and SIR error. The authors assume that interference level does not change significantly from one measurement to the next and are slower that the power updates time-scales. Simulation results have demonstrated the superiority of the proposed controller to the power balancing algorithm in minimizing power usage and SIR error.

\subsection{Summary}

In summary, most of the DTPC algorithms studied in literature are predictive in order to reduce the control system delay. Based on the temporal correlation of interference and the SINR, previous works have exploited robust filters to estimate the channel state conditions. However, it has been inferred that base stations assign orthogonal CDMA codes or TDMA slots to mobile nodes in their cell or sector and a possible high transmission power level may be used. In spite of many decentralised control algorithms, most algorithms rely on the physical layer SIR measures for the optimal DTPC. However, according to Kumar and Kawadia [14], the DTPC algorithms are affected by a number of link state information (LSI) across the network protocol stack. We can argue that these LSI, too, do have temporal correlations and thus can be modelled using state space techniques solvable by predictive approaches. If the overall WBMN is considered to comprise of a large number of interconnected distributed control loops, then methods from control theory may be used to assess stability and dynamic behaviour of the network. Thus, the use of dynamic control theoretic algorithms may an interesting area of future topic.

\section{Power Controlled Network Protocol Heuristics}

Fig. 4 illustrates a single transmission from node $\mathrm{A}$ to node $\mathrm{B}$ on a co-channel environment with other nodes of the network. Table 1 provides a motivation behind such illustrations. If node A chooses a minimum power level (i) then significant power saving and better spatial reuse may be achieved but hidden terminal problems (HTPs) around node B may aggravate the network throughput. If node A uses a power level (ii) then not much power saving and network fairness can be achieved. On the other hand, if node A chooses a maximum power level (iii) then all HTPs is resolved but there is inefficient spectrum reuse and energy consumption and thus may aggravate network throughput (capacity) even further. Therefore, if node A uses a "proper” power level (iv) then a fair trade-off between power and network capacity may be attained. Allocating the transmission power level dynamically on a per-packet basis requires the cross-layering information [14]. This area has widely been 
studied for the general wireless Mobile Ad Hoc and Sensor Networks but missed specialized attributes of the WBMNs. This section outlines studies relevant to the WBMNs context and highlights open issues.

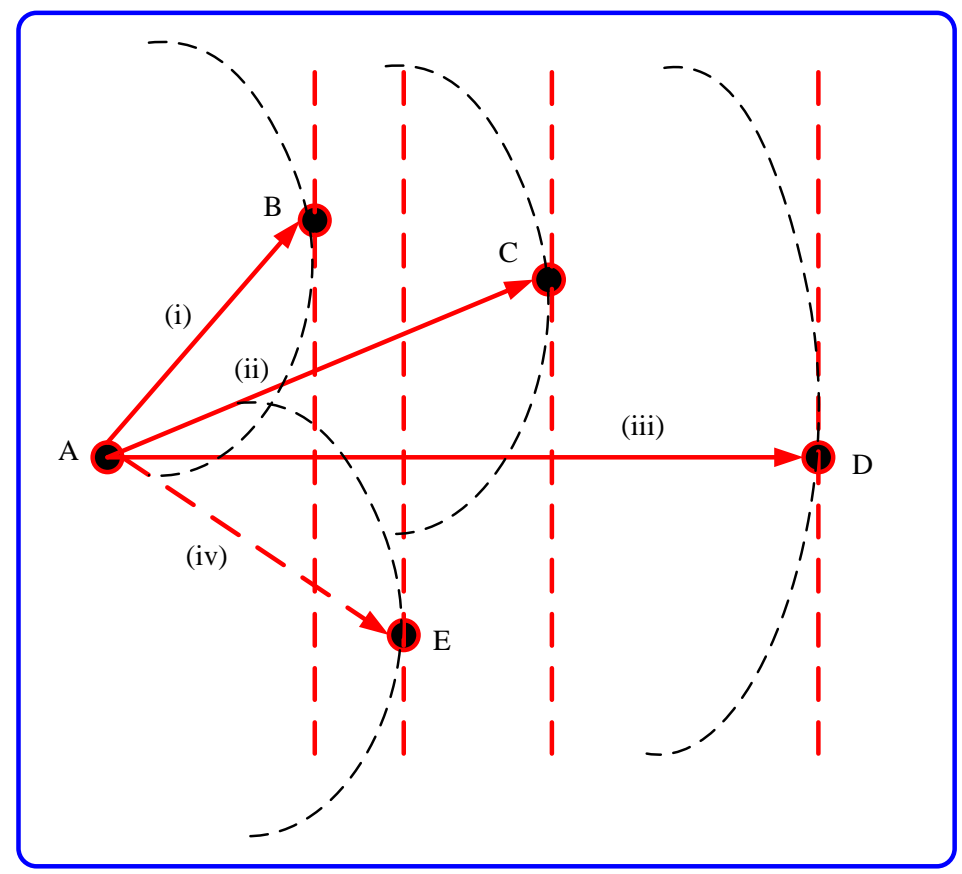

Figure 4: Motivating DTPC (node A to node B) for Single Channel Networks

Table 1. Summary of TPC motivation

\begin{tabular}{|c|c|c|c|}
\hline \multicolumn{2}{|l|}{ Action } & Advantages & Disadvantages \\
\hline (i) & A-B at Minimum Power & Save power and better spatial reuse & $\begin{array}{l}\text { Maximum HTPs e.g., contention and } \\
\text { collisions and aggravated network } \\
\text { throughput }\end{array}$ \\
\hline (ii) & $\begin{array}{l}\text { A-C at Some power }>\text { Min } \\
\text { power }\end{array}$ & $\begin{array}{l}\text { Some power saving, Some fairness, some } \\
\text { spatial reuse }\end{array}$ & \\
\hline (iii) & A-D at Maximum power & $\begin{array}{l}\text { Interference Avoidance i.e., suppressed } \\
\text { HTPs }\end{array}$ & $\begin{array}{l}\text { Poor spatial reuse, Inefficient power use } \\
\text { and Bandwidth unfairness }\end{array}$ \\
\hline (iv) & $\begin{array}{l}\text { A-E at properly controlled } \\
\quad \text { power }\end{array}$ & $\begin{array}{l}\text { Optimal saving, Optimal network capacity } \\
\text { and Optimal fairness }\end{array}$ & \\
\hline
\end{tabular}

\subsection{Protocols Based on Omni directional-MAC}

The role of the medium access control (MAC) protocol is to modify the transceiver parameters (e.g., transmission power level and antenna beams) or radio resource (e.g., channels) or the topology of a network. At MAC level the aim is to reduce energy consumption, improve throughput or both. Our focus in this review lies on how the MAC protocol adjusts the transmission power level dynamically for every packet transmitted. This section covers only central contributions in this area relevant to the static WBMNs. 


\subsubsection{Common Channel Based}

In [84], Cheng et al., have reviewed the distributed MAC protocols in terms of the objectives and methodology as applied to the resource allocation problems in the WMNs. Research on varying the transmission power level on a per-packet basis in Ad Hoc networks have been thoroughly conducted by [85]. In the conventional way, maximum transmission power level is used for request-to-send (RTS) and clear-to-send (CTS) packets while the minimum required transmission power is used for data and acknowledgement (DATA-ACK) packets in order to save energy [29]. However, RTS-CTS packet exchanges reserve a large floor space for DATA-ACK conversations thereby degrading the spatial reuse by other network users. In order to eliminate hidden terminal problems (HTPs), [85, 86] have introduced a power controlled MAC (PCM) protocol. In PCM, RTS-CTS packets are transmitted at maximum power and data is periodically also transmitted at maximum power; the data is otherwise sent at optimal power to conserve energy. The periodic maximum power for data transmissions enable nodes in the sender's carrier sense zone to stay suppressed throughout the sender's transmission. This asymmetric transmission power results in unfairness of medium access among users. In fact, transmission power control increases the number of hidden terminals in the network and exacerbates the unfairness of the medium access for less powerful links in the network. SHUSH technique proposed by Sheth and Han [87] tackles unfairness problem among the low and high transmission power nodes. The SHUSH technique suggests a reactive transmission power control for wireless MAC protocols. SHUSH is grafted into the reactive strategy of SHUSHing the interferer. Only after there is interference do nodes react by identifying the source of interference via the header fields in the interfering packets. Nodes initiate the control packets at optimal power needed to reach and SHUSH the interferer. The DATA-ACK packets are exchanged also at optimal transmission powers. Autonomous power adjustment for RTS-CTS packets in addition to that of the DATA-ACK packets has been well studied by Chen et al., [86].

Muqattash and Krunz [31] have proposed POWMAC: a single-channel power-control protocol for throughput enhancement in wireless Ad Hoc networks [88-90]. Instead of alternating between the transmission of control (i.e., RTS-CTS) and data packets, as done in the 802.11 scheme [88], the POWMAC uses an access window (AW) to allow for a series of RTS-CTS exchanges to take place before several concurrent data packet transmissions can commence. The length of the AW is dynamically adjusted based on localised information to allow for multiple interference-limited concurrent transmissions to take place in the same vicinity of a receiving terminal. Simulation results demonstrated that significant throughput and energy gains are obtainable with POWMAC protocol. However, it is difficult to implement synchronization between nodes during the access window (AW). The POWMAC does not solve the interference problem either. Siam and Krunz [90] have enhanced the throughput and energy performance of the POWMAC and the IEEE 802.11 standard protocols. They have integrated multi-input multi-output (MIMO) capabilities to these protocols and noted that the MIMO systems double the bit rate per link over the single-input single-output (SISO) systems (i.e., use of single antenna). Throughput gains in the MIMO systems are observed at a non-negligible energy costs. However, MIMO systems are not energy efficient techniques albeit. 
Ding et al., [91] have proposed a per-frame-based transmission power control protocol, DEMAC in IEEE 802.11 at low PHY rate. The aim is to avoid network interference, improve throughput and save energy. In DEMAC, transmit power of RTS is used to find the interference in the network. The receiver calculates the optimal transmit power for the data frame based on the data payload and the current interference. CTS, DATA and ACK would then be transmitted with this optimal transmit power. Although DEMAC avoids network interference at both the sender and receiver side, yields a good throughput and energy saving, it does not take into account high PHY rate. The DEMAC employs maximum power with RTS packets and thus poor spatial reuse. Jia et al., [92] have improved on the work by Ding et al., [91] by providing a scheme that carefully combines collision avoidance and spatial reuse for IEEE 802.11 MAC protocols. Simulation results showed up to 40\% throughput increase and 3 times more data delivery with the same amount of energy under single-channel, single-transceiver design conditions. Although the authors have provided theoretical analysis to justify the scheme and simulation results, interference avoidance and fairness was assumed only at the receiver side. Li et al., [93] proposed adaptive transmission power control protocol (ATPMAC) which can enable several concurrent transmissions without interfering with each other while incurring minimal signalling overhead cost. Considering the single channel and single transceiver nodes Ad Hoc networks, ATPMAC has shown up to $136 \%$ throughput improvement compared to the IEEE 802.11 in a random topology. The method, however, does not take into account interference effects at the sender side [94].

In order to allow fairness, Ho and Liew [95, 96] have proposed and investigated two distributed adaptive power control algorithms that minimize mutual interference among links while avoiding the hidden nodes [97]. Hidden nodes cause unfair bandwidth distributions and bandwidth oscillations. The two algorithms adapt the transmission power of each transmitter link to the positions of its surrounding links in addition to the connectivity requirements with its receiver link. The algorithms demand that: links that do not mutually interfere with one another remained non-interfering while existing interfering links can be made non-interfering after the transmission power adjustment. Also, no new hidden nodes will be created. Through extensive experiments, the authors observed that the proposed algorithms achieve a good balance between the scalability of the network capacity (which is related to the exposed node) and fairness (which is related to the hidden node) in wireless networks [98].

In [11], Correia et al., performed experiments on two dynamic transmission power adjustments in MAC protocols for wireless sensor networks (WSNs). The first approach employs dynamic power adjustments by exchanging information among nodes, and the second one calculates the ideal transmission power according to signal attenuation in the link. Both methods used a clear channel assessment (CCA) mechanism (i.e., which does not exploit channel reservation, e.g., RTS-CTS messages). Nodes periodically sample the signal strength if there are ongoing transmissions in order to determine the maximum noise level (base noise). If the sampled signal strength is higher than the base noise, then the protocol detects ongoing transmission and that transmission power is dynamically and accordingly adjusted by the node [99]. The CCA mechanism has the advantage of mitigating collisions over the reservation based schemes. In another paper, Correia et al., [100] have extensively discussed the benefits of the transmission power control MAC protocols, examined the issues 
in the implementation of such protocols and summarized the results of their first evaluation using existing hardware [26].

In [71], authors have established an effective transmission power control mechanism by understanding the dynamics between link qualities and received signal strength indicator and link quality indicator (RSSI/LQI) values. They have presented empirical results that demonstrate the relation between the link layer quality and RSSI/LQI. It has been observed that, Radio Irregularity results in radio signal strength variation in different directions, but the signal strength at any point within the radio transmission range has a detectable correlation with transmission power in a short time period. A similar empirical experiment was studied by Jeong et al., [101]. Authors based their work on previous studies and evaluated power control algorithms using realistic multi-hop WSN workloads and a large Mica2dot based test-bed. The experimental results have indicated that the dynamic power control MAC protocol with low duty cycles yields up to $16 \%$ more power saving than the fixed power controlled counterparts.

\subsubsection{Separate Channels Based}

From a different perspective, Dongsheng et al., [28] have emphasized on the need to combine both power control (i.e., to reduce energy consumption and increase spatial reuse) and least interference channel assignment (i.e., to reduce collisions and improve fairness) and to combine both the MAC layer and the network layer (i.e., to guarantee network connectivity and eliminate frequent topology changes). This research has been triggered by the fact that the transmission power control by use of minimum power not only reduces energy consumption but also brings more collisions and frequent changes in topology. In order to solve such problems, the authors have proposed a protocol with the following features: independent channels are used to transfer data packets to reduce collisions in data transmissions, Control packets are transferred at maximum power and data packets are transferred at proper power in order to reduce energy consumption, guarantee network connectivity and eliminate frequent topology changes. However, the use of maximum power to eliminate HTPs in turn aggravates the spatial reuse and thus poor network capacity.

In order to reduce collisions further, [7, 8, 102] combined the carrier sense multiple access (CSMA) with a spread spectrum-code division multiple access (SS-CDMA) which does not require the central controller [7]. They have observed that through the use of local coordination only, packet collisions were completely avoidable by employing the hybrid MAC protocol and power control. A better trade-off between bandwidth usage and latency could also be noted compared to IEEE 802.11 MAC. The authors have assumed a decentralized SS-CDMA but have not investigated further how codes are spread across the network and with how much power level.

It has been well argued that minimizing the transmit power (i.e., to eliminate exposed node problem (ENP)) not only improves spectral reuse and reduces energy consumption but also introduces HTPs [84]. In order to eliminate HTPs, Lai et al., [103] have presented power control interference avoidance (IA) scheme. The authors have shown a proper way to adjust the transmission power of the control packets and data packets such that large interference's range problems are resolved and low energy is consumed comparative to IEEE 
802.11. This is achieved by first dividing the channel into control channel and data channel. The RTS, CTS and ACK packets are sent in control channels and DATA packets are sent in data channels. The RTS and DATA packets are then sent with a smaller transmission power (i.e., a tenth of the maximum power) and CTS and ACK are sent with a larger power (i.e., the maximum power) in order to suppress the interferers within the vicinity of the receiver [103]. However, they have not estimated interference within the vicinity of the transmitter during control signalling exchanges.

In order to utilize the radio spectrum efficiently and resolve potential contention among nodes using the medium, multi-channel and power control MAC protocols are used jointly [104]. The added benefits are that while multi-channel provides an improved network performance as the number of the nodes causing contention and collision increases, the transmission power control provides spatial reuse and minimizes energy consumption. If a node is empowered to access multiple channels such as in CDMA technology, then a node can utilize multiple codes simultaneously or dynamically switch from one code to another as needed. In this way, authors have achieved the channel assignment, multiple access and power control solutions in an integrated framework [105-107]. It should however, be noted that assigning channels, estimating cross-layer states and performing the DTPC may cause system delay problems. Multi-radio configurations have been designed to resolve system delay problems [108]. However, no much work exists in the literature for the multi-radio DTPC problems.

\subsubsection{Summary}

In summary, the above power-controlled MAC protocols assume that nodes have equal reception sensitivity and radiate equal power in all directions. The argument behind this assumption is that if any node can cause interference at a receiver then it will most likely hear the CTS from that receiver and defer from transmitting. However, when directional antennas are used, the radiated power and reception sensitivity between any two nodes become a function of the angular orientation of these nodes. Thus, using omni-directional power for RTS-CTS and data packets, even at different levels, can no longer prevent all potential interferers from transmitting [109]. However, when directional antennas are employed without invoking proper power control, typical MAC protocols for the IEEE 802.11 Ad Hoc mode can face several medium access problems. For example, interference from minor lobes and HTPs may cause medium access unfairness [110]. The issue of accessing and forwarding traffic simultaneously while maintaining bandwidth fairness through power controlled multiple channel MAC protocols, remains an open area of research.

\subsection{Protocols Based on Directional-MAC}

The use of directional antennas offers wireless Ad Hoc network, numerous benefits. That is, extended communication range, better spatial reuse, capacity improvement, and suppressed interference [109]. Numerous studies exist in this area but we give most of central contributions to the WBMNs.

Capone and Martignon $[18,111]$ have argued that while adaptive antennas can improve 
the utilization of the wireless medium (i.e., increased network capacity) by reducing radio interference and the impact of the exposed node problem, it can exacerbate the HTP [27]. As a result information about wireless medium reservation needs to be spread to the maximum possible extent without interfering with the connections already established in the network. In order to achieve an improved total traffic accepted by the network (i.e., capacity) and fairness between competing connections, Capone and Martignon [18] have proposed a scheme in which RTS-CTS frames are sent in all antenna sectors at the maximum allowed power that does not cause interference with the ongoing transmissions. The DATA-ACK packets' exchange then takes place directionally with the minimum necessary power. Instead of using maximum power for control traffic, one should employ power efficient antenna radiation gain.

Arora et al., [109] suggested a power-controlled MAC protocol for directional antennas that ameliorates HTPs and improve energy-saving [27]. The protocol used separates control and data channels to reduce collisions. It allows for the dynamic adjustment of data-packet transmission power such that this power is just enough to overcome interference at the receiver. In [27], Arora and Krunz have proposed load control access protocol (LCAP) that allows transmissions to take place along already reserved directions provided that the SINR at the receiving nodes remains above the predefined SINR. The proposed LCAP manages throughput and energy trade-off in a power-controlled (interference-limited) wireless communications with directional antennas. The LCAP are categorised into two types. Interference-based LCAP and the overall throughput based LCAP. In the former, when a new user is to be admitted, the service provider estimates the expected total interference due to the addition of this new user. The increase in the interference depends on the user's quality of service (QoS) requirements (bit rate, required bit-error-rate, etc). The new user is admitted only if the total expected interference is below a predefined threshold. In the latter case, the prospective user is not admitted if the total normalised throughput following the admission is expected to exceed a predefined threshold. The simulation results have demonstrated that the combined gain from concurrent transmissions using directional antennas and power control provides a good trade-off between network throughput and energy consumption.

In [112], Alawieh et al., have analysed the benefits of transmission power control on throughput and energy consumption in Ad Hoc networks with directional antennas. First the authors have constructed an interference model for directional antenna based on honey grid model to calculate the maximum interference [113]. They have then developed a collision avoidance model [114]. The authors have also presented the maximum end-to-end throughput under the maximum interference constraints. It has been noted that by selecting a smaller carrier sense threshold (i.e., interference range) will severely impact on the spatial reuse where as a larger carrier sense threshold will yield excessive interference among concurrent transmissions [115]. Thus, a need for an appropriate selection of the carrier sense threshold so that power control can reduce collisions significantly.

\subsubsection{Summary}

In summary, the use of directional antennas and performing power control allows improved throughput and energy-saving since they have higher gain over their equivalent 
omni-directional counterparts. However, frequent beam switching to specific target receivers may turn out to be energy-inefficient [113]. To access and route traffic simultaneously, sectored antennas driven by power controlled dual-band radios or soft-ware defined radios may be desirable $[25,108]$. This area requires much research attention.

\subsection{Protocols Based on Power Management}

In order to sustain long lifetimes of wireless networks on limited energy resources two major approaches have been proposed: transmission power control and sleep schedules. While transmission power control reduces the radio power consumption in the transmission state, the sleep scheduling reduces the radio power consumption in the idle state by turning off radios when not in use. Thus integration of the two is often referred to as Power Management [116]. Detailed taxonomy of power management protocols for wireless networks have been provided by Zheng et al., [117]. Numerous DTPC contributions exist in this context for mobile Ad Hoc and WSNs. We outline works that fit into attributes of WBMNs.

Jamieson et al., [118] have proposed a power-saving technique for multi-hop Ad Hoc wireless networks called Span. Span has been based on the observation that when a region of a wireless Ad Hoc network has a sufficient density of nodes, only a small number of them need to be on at any time to forward and coordinate traffic for active connections. Experimental design has proved that the system lifetime of a Span 802.11 network is a factor of two better than a Standard 802.11 network. Span yields an improved latency and competitive bandwidth. Xu et al., [119] presented two topology control protocols that extend the lifetime of dense Ad Hoc networks while preserving connectivity, the ability for nodes to reach each other. The methods conserve energy by identifying redundant nodes and turning their radios off. The first method is the Geographical Adaptive Fidelity (GAF), which identifies redundant nodes by physical location and a conservative estimate of radio range. The other one is the Cluster-based energy conservation, which directly observes radio connectivity to determine redundancy and so can be more aggressive to radio fading. Analysis, simulation and experimental tests have shown that both protocols are robust to node failures, radio propagation and node deployment density. However, these proposals have focussed on the network operations and neighbour discovery, Hsu and Hurson [120] have applied probabilistic wake-up based power management to neighbour monitoring which aims to reduce energy consumption while preserving the effectiveness of misbehaving node detection.

An asynchronous power save protocol has been discussed in depth by [121]. In this protocol, neighbours that wish to communicate, estimate their relative phase difference between their sleep and wake cycles. A station then uses this phase information to transmit its pending packets over the available periods most efficiently. The advantage is that, stations can adjust their phase relationships to avoid contention and reduce latency for delay sensitive flows. In a recent study Feeney et al., [122] have investigated the impact of wakeup schedule distribution in asynchronous power save protocols on the performance of multi-hop wireless networks. The asynchronous wakeup schedules create an uncoordinated pattern of times at 
which nodes will attempt to transmit. Simulation data showing that the capacity associated with the best wake-up patterns is significantly larger than that of the worst. The result gives an insight to the behaviour of such protocols and acts as a feasibility study showing the potential benefit of mechanisms by which nodes adapt their wake-up schedules to obtain improved performance.

In stead of placing inactive components into low power states using a single dynamic power management (DPM) policy, Dhiman and Rosing [123] have suggested a selection among a set of DPM policies using machine learning algorithm. This algorithm adapts to changes in workloads and guarantees quick convergence to the best performing policy for each work load. In a similar spirit, unified power management architecture (UPMA) for supporting radio power management in WSNs is provided in [124, 125]. The UPMA provides: a set of standard interfaces that allow different sleep scheduling policies to be easily implemented on top of various MAC protocols at the data link layer and an architectural framework for composing multiple power management policies into a coherent strategy based on application needs. Minimum power configuration that minimizes the aggregate radio power consumption of all ratio states has been suggested by Xing et al., [126]. However, in both [125] and [126], the transmission power choices and the sleep scheduling decisions of nodes are coordinated according to the current network workload. The UPMA enables cross-layer coordination and joint optimization of different power management strategies that exist at multiple network layers while allowing them to have independent implementations [127].

Zheng and Kravets [128] have proposed an on-demand power management framework for multi-hop wireless networks. In this framework, power management decisions are driven by data transmission in the network. Nodes maintain soft-state timers that determine power management transitions. By monitoring routing control messages and data transmissions, these timers are set and refreshed on demand. Only nodes on the communication path along which a connection is routed are kept active while all the other nodes can switch to the power-save mode. The benefit achieved is that soft states are aggregated across multiple flows and its maintenance required no additional out-of-band messages. However, no incentive has been given to keep some nodes active while others sleep. Motivated by the observation that explicit and periodic re-computation of the backbone topology [119] is costly with respect to its additional bandwidth overhead, a probabilistic power management has been constructed by $\mathrm{Li}$ and $\mathrm{Li}$ [129]. The authors believe that any schemes involving periodic and local broadcasts of messages do not scale well as the number of node density increases. Since each node needs to broadcast a message during each broadcasting interval, as the number of nodes increases, such broadcasts will eventually saturate the residual capacity of the network thereby causing collisions and disruptions to the ongoing data traffic. The contributions of probabilistic (Odds) approach are that, it guarantees network scalability, uninterrupted ongoing traffic, network parameter flexibility and compatibility with IEEE 802.11 MAC layer standard. However, Odds suffers from heavyweight computations, a prime consumer of the network energy. Odds have not shown whether or not an optimal transmission power is used for selected active nodes.

Zhang et al., [130] have proposed a time-division multiple-access (TDMA) based 
multi-channel MAC protocol called TMMAC in order to save power for Ad Hoc networks. TMMAC requires only a single half-duplex radio transceiver on each node. In addition to explicit frequency negotiation, TMMAC introduces a lightweight explicit time negotiation in order to exploit the advantage of both multiple channels and TDMA so as to set inactive nodes to doze mode. The simulation evaluation has shown $113 \%$ higher communication throughput and $74 \%$ less per packet energy over the state-of-the-art multi-channel MAC protocols for single transceiver wireless devices. The drawback of TMMAC is that time negotiations are difficult to distribute across the network without incurring bandwidth overheads. While single half-duplex radio transceiver operations are energy-efficient, such configurations coupled with multiple channels require sophisticated switching and thus exacerbates delay [131].

Wang et al., [131] considered the joint design of opportunistic spectrum access (i.e., channel assignment) and adaptive power management for multi-radio multi-channel wireless local area networks (WLANs). Their motivation has been the need to improve throughput, delay performance and energy efficiency [132]. In order to meet their objective, they have suggested a power-saving multi-channel MAC (PSM-MMAC) protocol which is capable of reducing the collision probability and a wake state of a node. The design of the PSM-MMAC relied on the estimation of the number of active links, queue lengths and channel conditions during the ad hoc traffic indication message (ATIM) window [133]. Through numerous simulation results, a good trade-off was observed for throughput, delay performance and energy-efficiency compared to the previous approaches [133, 134].

\subsubsection{Summary}

In summary, the power control by alternating dormant state and transmission state of a transceiver is an effective means to reduce the power consumption significantly. However, most of previous works have emphasized that wake-up and sleep schedule information are distributed into the network. The overhead costs associated to this have not been thoroughly investigated. Furthermore, transmission powers for active connections have not been guaranteed optimal. This aspect should be taken into account in future studies.

\subsection{Protocols/Algorithms Based on Connectivity Management}

In order to attain desirable network connectivity attributes: minimal average node degree (e.g., [135, 136]), fault tolerant connectivity [137, 138] and spanner network structure (e.g., [36, 139]), topology control algorithms (TCAs) using optimal transmission power adjustment (TCATPA) have been studied (e.g., [136, 140, 141]). The topology of a multi-hop wireless network is the set of communication links between node pairs used explicitly or implicitly by a routing mechanism. The WBMN topology is affected by many factors: weather conditions, medium objects, interference, channel noise, battery power outage, the power levels and antenna radiation directions. Of importance, is to adjust the transmission power level of each node dynamically based on the desired connectivity attributes. In Fig. 5, a connection from node A to node B with transmission power levels (i), (ii), (iii) and (iv) may result in a dis-connected, a fully connected, a critically connected and a 'properly' connected network, 
respectively. Table 2 provides a summary of advantages and disadvantages of choosing various transmission power levels (i.e., homogenous ranges). The heterogeneous network is a generic model of this homogenous scenario.

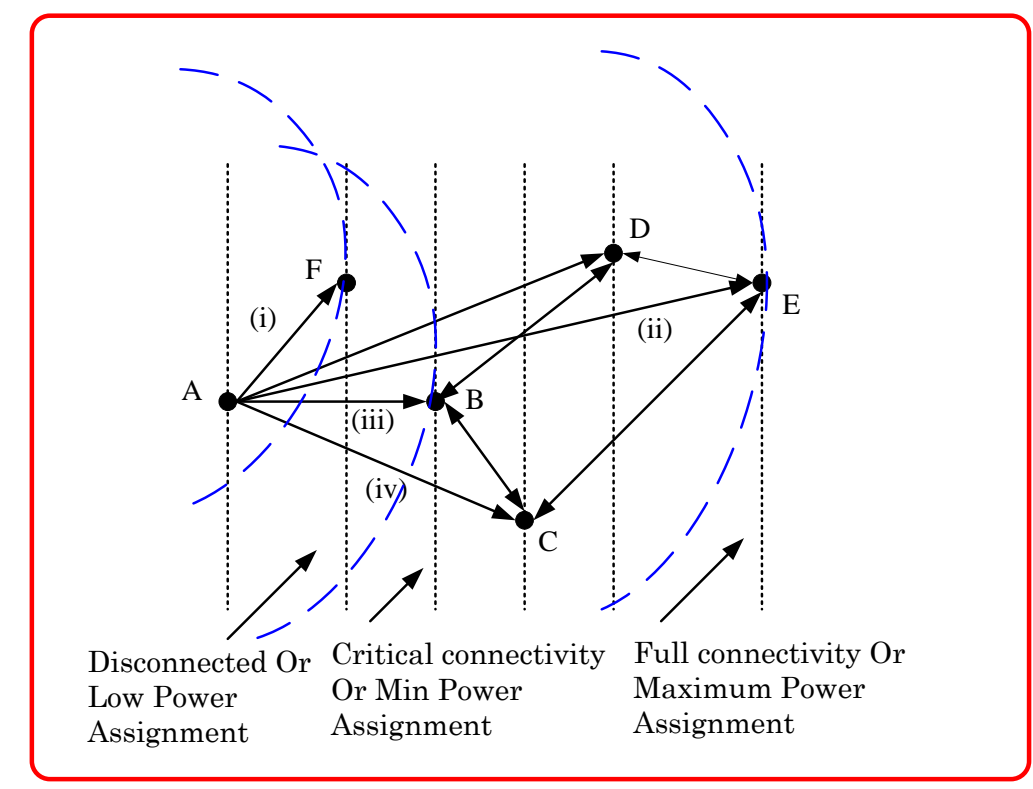

Figure 5: Connectivity Range Adjustment for node A to node B: Homogenous case.

Table 2. Summary of Topology Control based DTPC Algorithm

\begin{tabular}{|c|c|c|c|}
\hline & Action & Advantages & Disadvantages \\
\hline (i) & A-F, Low connectivity range & $\begin{array}{l}\text { Significant power saving and Enhanced } \\
\text { multi-hop routing (fault-tolerance) }\end{array}$ & $\begin{array}{l}\text { Node A and B are disconnected directly by } \\
\text { one hop and Routing delays }\end{array}$ \\
\hline (ii) & $\begin{array}{l}\text { A-E, Maximum connectivity } \\
\text { range }\end{array}$ & $\begin{array}{l}\text { Fully connected network and user-specific } \\
\text { Quality of Service (QoS) improvement e.g., } \\
\text { reduced user latency }\end{array}$ & $\begin{array}{l}\text { Very high energy consumption and thus } \\
\text { short network life, very high node A degree } \\
\text { e.g., F, B, C, D and F, and an increased } \\
\text { congestion to other nodes }\end{array}$ \\
\hline (iii) & A-B, Critical connectivity & Power saving and some network capacity & $\begin{array}{l}\text { Critical resilience to topology changes, } \\
\text { dominant unfairness from hidden nodes }\end{array}$ \\
\hline (iv) & $\begin{array}{c}\text { A-C, properly adjusted } \\
\text { connectivity range }\end{array}$ & $\begin{array}{l}\text { Minimum average node A degree e.g., F, B } \\
\text { and C, } \\
\text { enhanced multi-hop, high spatial reuse and } \\
\text { high network capacity }\end{array}$ & $\begin{array}{l}\text { Fair energy loss and suppression to } \\
\text { immediate neighbours thus, introducing } \\
\text { some unfairness }\end{array}$ \\
\hline
\end{tabular}

Numerous studies on the DTPC based on the TCA for distributed wireless networks have been conducted (e.g., [140-142]). In [136], Ramanathan and Rosales-Hain studied the problem of adjusting transmit powers of nodes to create a desired network topology. The authors formulated the transmit power adjustment problem as a constrained optimization problem. In this case, the connectivity and bi-connectivity were taken as constraints and the maximum power used as an optimization objective. They suggested the so called Connected MinMax Power (CMP) and Biconnectivity Augmentation with MinMax Power (BAMP) 
problems. These problems assumed a given multi-hop wireless network, $M=(N, L)$, where $N$ is a set of nodes in the entire network and $L: N \rightarrow\left(Z_{0}^{+}, Z_{0}^{+}\right)$is a set of coordinates on the plane denoting the location of the nodes. Specifically, the CMP problem finds a per-node minimal transmit power assignment $p: N \rightarrow Z^{+}$, such that the induced graph of $(M, \lambda, p)$ is connected, and $\operatorname{Max}_{u \in N}\left(p_{u}\right)$ is minimum. The induced graph is represented as: $G=(V, E)$, where $V$ is a set of vertices corresponding to nodes in $N$, and $E$ is a set of undirected edges such that $(u, v) \in E$ if and only if $p_{u} \geq \lambda(d(u, v))$, and $p_{v} \geq \lambda(d(u, v))$. The BAMP

Problem tries to find a per-node minimal set of power increases $\delta(u)$ such that the induced graph of $\left(M, \lambda, p_{u}+\delta(u)\right)$ is bi-connected, and $\operatorname{Max}_{u \in N}\left(p_{u}+\delta(u)\right)$ is minimum. In order to solve these problems, the authors proposed two centralised algorithms for static networks and two distributed heuristics for mobile networks. This work minimized the maximum transmission power rather than the total power to maintain system scalability.

Wattenhofer et al., [138] have suggested a transmission power adjustment scheme related to the approach in [136]. The authors have shown that each node makes local decisions about its transmission power and these local decisions collectively guarantee global connectivity. Specifically, a node receiving directional information grows its transmission power gradually until it finds a neighbour node in every direction. Simulation results have shown that with low transmission power adjustment and low node degree attainment, the network lifetime is increased and traffic interference is reduced [143]. Tan and Seah [143] have proposed the critical neighbour (CN) scheme, which adaptively adjusts the transmission power of individual nodes according to route and traffic demands, to reduce the level of interference amongst nodes in the network. Simulation results have indicated that the CN scheme has higher throughput and lower end-to-end delay than the unmodified version.

Li et al., [142] have studied the strong minimum power restricted topology control problem in wireless sensor nodes. This is to adjust the limited transmission power for each wireless node and to find a power assignment that reserves the strong connectivity and achieves minimum energy cost in the wireless nodes. Three heuristics based on the problem have been proposed. Simulation results have demonstrated the efficiency of the heuristics.

Calinescu et al., [144] have reported analytical power assignment problems. In Calinescu et al., [145] gave approximation algorithms for the Min-Power Symmetric Connectivity, Min-Power Strong Connectivity and Min-Power Broadcast. They also considered a special treatment for the important case of Power Symmetric Connectivity in the Euclidean with node-dependent transmission efficiency. In [144], they analysed the assignment and concluded that adjusting transmission power can present strong network connectivity.

Wang and $\mathrm{Li}$ [36] have generalized the work in [136, 138]. They have addressed the problem of finding minimum power assignment of each individual node such that the 
undirected induced communication graph is a spanner of the communication graph when all nodes transmit at their maximum power. The term induced undirected communication graph is defined by an edge $u v$ if and only if $p_{u v} \leq p_{u}$ and $p_{u v} \leq p_{v}$ with $p_{u}$ and $p_{v}$ as power settings for node $u$ and $v$, respectively. However, if all wireless nodes transmit with their maximum power $p_{\max }$ then the induced communication graph is called the original communication graph, or simply a unit disk graph (UDG). It should be noted that at maximum power, each node may have information about all possible network topologies. The term, a spanner, means that the length of the shortest path in the induced communication graph is at most some constant multiplied by the length of the shortest path in the original communication graph. However, the minimum power assignment does not always guarantee the minimum average node degree in spite of a strongly connected network [36].

The authors in [36], have developed a polynomial time algorithm that minimizes the maximum assigned power. They have also presented a polynomial time approximation method to minimize the total transmission radius of all nodes. The algorithm and approximation are based on two heuristics. The simulation studies conducted to verify their efficacies. However, the minimization of the total transmission radii implies that each node has global knowledge of the network at the expense of communication overheads. The approach has a little practical value since battery power-life is localized. An energy-efficient extension to the work by [36] has been found in [139]. Khan et al., [139] and Choi et al., [146] presented a distributed construction of the minimum spanning tree (MST) problem. This problem was formulated as a geometric weighted MST problem. That is, given an arbitrary set $N$ of nodes in a plane, find a tree $T$ spanning $N$ such that $\sum_{(u, v) \in T} d^{\alpha}(u, v)$ is minimized, where $d(u, v)$ is the distance of an edge $(u, v) \in T$

according to the Euclidean norm and $\alpha=[2,4)$ depicts environments with multiple-path interference or local noise. Khan et al., identified that in Kruskal's algorithmic construction, the MST which minimizes $\sum_{(u, v) \in T} d(u, v)$ also minimizes $\sum_{(u, v) \in T} d^{\alpha}(u, v)$ for any $\alpha>0$. In order to solve the distributed MST problem, the authors developed the so called the Nearest Neighbour Tree (NNT) algorithms with low energy complexity. The NNT operates on the idea that each node independently chooses a unique rank, a quantity from a totally ordered set, and that node connects to the nearest node of higher rank. This precludes overhead cycles with the edges already chosen. The only information that needs to be exchanged is the rank. However, the procedure on how to evaluate the set of ranks has not been provided.

Gerharz et al., [147, 148] introduced a simple distributed algorithm that assigns individual transmission powers to wireless devices. The authors proposed a cooperative nearest neighbour topology (CNNT) control strategy. Each device is assigned the low transmission power that connects it to the $k$-nearest "neighbours". Node $A$ is the neighbour of 
a node $B$ if and only if there is a bidirectional link between $A$ and $B$. Through simulations, the topologies created by CNNT algorithm without any global knowledge are as effective as topologies resulting from a good choice of a common transmission power (which would require global knowledge) in terms of the achievable throughput. The CNNT was thus an improvement of NNT. However, common transmission power adjustment does not only cause excessive message overhead across the network but also assumes prior that the network nodes are uniformly distributed.

$\mathrm{Li}$ and Hou [149], have proposed two localised topology control algorithms for a general wireless networks with each node having different maximal transmission power. The two algorithms are: the Directed Relative Neighbourhood Graph (DRNG) and the Directed Local Spanning Sub-graph (DLSS). In both algorithms, each node independently builds its neighbour set by adjusting the transmission power and defines the network topology by using only local information. Authors have proved that both the DRNG and the DLSS can preserve network connectivity, the out-degree of any node in the resulting topology generated by the DRNG or DLSS is bounded by a constant and the DRNG and the DLSS can preserve network bi-directionality.

Aron et al., [140] have considered the problem of topology control for hybrid wireless mesh networks (WMNs) with heterogeneous. A localised distributed topology control algorithm was developed which calculates the optimal transmission power so that: network connectivity is maintained, node transmission power is reduced to cover only the nearest neighbours and network lifetime is extended. These goals have been confirmed through numerous simulation tests. However, mobile mesh clients have not been considered in spite considering the hybrid mesh architecture. In other works, Aron et al., [150, 151] have proposed the local minimum shortest-path tree (LM-SPT) and/or the local neighbourhood shortest-path tree (LN-SPT) algorithms suited for WBMNs. The algorithms are distributed with each node using only the information gathered locally to determine its own transmission power. The implementation follows two phases. The first phase is to construct a minimum local or neighbourhood shortest-path tree. The other phase is to gradually remove all unidirectional links. Authors have performed several simulation tests and noted that the resultant network topology preserved the network connectivity, reduced the average node degree, ensured evenly distributed power consumption among nodes and reduced significantly the total power consumption leading to longer network lifetime [119].

In a similar framework to the work in [146], Bhatia et al., [135] have investigated a power balancing problem in energy constrained multi-hop wireless networks. The objective is to minimize the maximum average power used by the nodes such that no node uses much more power than others. The authors have formulated the problem of two power assignment under the constraint that the network connectivity is maintained. However, such a problem has been shown to be an NP-hard and also hard to approximate [138]. Because finding the maximum average power is not feasible with a fully decentralized algorithm. The distributed localised heuristics proposed to solve this problem have shown that the algorithm can reduce the average power significantly when compared with algorithms that assign a common power [147]. However, excessive message overhead costs across the network remained un-resolved by [135]. 
Li et al., [152], proposed a distributed mechanism to build a sparse power efficient network topology for non-uniform Ad Hoc wireless networks. In order to achieve their goal, authors extended the Yao structure [153] to build a spanner with a constant length and power stretch factor for mutual inclusion graph. They then suggested two efficient localised algorithms to construct connected sparse network topologies. They found out that both algorithms have communication cost $O(n)$, where each message has $O(\log n)$ bits. The proposed structure requires the use of maximum power in a sparse topology and thus energy-inefficient technique.

\subsubsection{Summary}

It is worthy concluding that a decentralized power adjustment is useful in topology control problems. Topology control problems are graph-theoretic problems. Optimal graph-theoretic methods, however, assume a global optimisation space otherwise are often NP-hard problems [138]. An optimal assignment of power by each node requires information across the network and thus overhead costs [136]. Furthermore, the unit disk graphs (UDGs) employed in the solution assume a 2-D plane or flat earth surface problem. However, the earth surface is composed of topographical obstructions. Many applications to structuring monitoring and underwater networks present a 3-D plane power control problem. The topology control based power control algorithms over-rely on the position of neighbour nodes in order to preserve the connectivity, maximum node degree, conserve energy and minimize interference. However, locations are complicated by several spatial factors [71]. In order to meet most of topology control stringent requirements, future work should consider localized range predictions to adjust transmission power level dynamically.

\subsection{Algorithms/Protocols Based on Joint Cross-layer Resource Management}

In their seminal paper, Kawadia and Kumar [14] remarked that power control in wireless networks is a cross-layer design problem. This is because the transmission power level affects signal quality and thus impacts the physical layer, determines the neighbouring nodes that can hear the packet and thus impacts the network layer, affects interference which causes medium access issues and congestion. Thus, impacts the medium access control and transport layers. Furthermore, power control aims at desirable throughput, delay and energy consumption performance $[19,26]$. Some of the power control jointly performed with cross-layer protocols will be reviewed in this section.

Wang et al., [154] have proposed a joint scheduling and power control algorithm, supporting multicasting traffic in a multi-hop wireless Ad Hoc networks. Multicasting enables data delivery to multiple recipients in a more efficient manner than traditional unicasting and broadcasting. A packet is duplicated only when the delivery path toward the traffic destinations diverges at anode, thus helping to reduce unnecessary transmissions (overheads). The algorithm performed an optimal power control based on [73] and connection scheduling when no optimal solution exists, i.e., how to determine which connections should not be allowed so that the admitted connections can enjoy a sufficiently high SINR. 
Muqattash et al., [89, 155] have proposed power controlled dual channel (PCDC) protocol that emphasizes the interplay between the MAC and network layers. They have described that the MAC layer indirectly influences the selection of the next-hop by properly adjusting the power of route request packets while maintaining network connectivity. Channel gain information obtained mainly from overhead RTS and CTS packets is used to dynamically construct the network topology. They have argued that unlike the IEEE 802.11 approach whereby RTS-CTS packets are used to silence the neighbouring nodes, collision avoidance information (CAI) can be inserted in the CTS packets and sent over an out of band control channel. The CAI is used to dynamically to bound the transmission power of potentially interfering nodes in the vicinity of a receiver [31]. Compared to the IEEE 802.11 approach, the proposed protocol has achieved a significant increase in the channel utilization and end-to-end network throughput, and a significant decrease in the total energy consumption. On a similar course, the authors have contributed towards a joint rate and power control with modulation adaptation $[53,156]$.

$\mathrm{Li}$ and $\mathrm{Wu}[157,158]$ have studied a power control and dynamic channel allocation for delay sensitive applications in wireless networks. In [157] they have investigated QoS-driven power allocation for the downlink of multi-channel, multi-user wireless networks. Authors have then proposed schemes based on multi-user and frequency diversities. Performance evaluations have shown that such schemes guarantee the QoS requirements compared to those which do not use multi-user diversity and power control. However, model approach confines to base-station based wireless cellular networks and thus limited applications [77]. Two phase distributed scheduling algorithm to identify a subset of wireless users whose QoS is guaranteed is found in [159]. In the first phase, each link transmits with a probing power and each user determines whether it can be a member of the basic feasible set or not in a distributed manner. In the second phase, a generalized call admission algorithm that attempts to merge as many as possible the rest links into the basic feasible set is developed. Through simulations, authors have evaluated the performance of the proposed scheme in terms of average execution time, average packet delay and maximum of the cycle time.

Narayanaswamy et al., [17] have studied theory, architecture, algorithm and implementation of the COMPOW protocol in wireless Ad Hoc networks. The COMPOW power control protocol for each node chooses a common power level, sets this power level to the lowest value which keeps the network connected and keeps the energy consumption close to the minimum. It has the property of ensuring bi-directionality of links due to reciprocity of electromagnetic waves in space. Authors have noted that the COMPOW protocol simultaneously satisfies three objectives of maximizing the traffic carrying capacity of the entire network, extending the battery life through providing low power routes, and reducing the contention at the MAC layer. The COMPOW protocol has the drawback that all links need to be symmetrical and all nodes must be homogenously distributed. This implies that even a single outlying node can cause every node to use a high power level. An improvement to the COMPOW protocol has been investigated by Bergamo et al.,[160]. In [160], each node estimates the power necessary needed to reach its own neighbours so as to yield energy efficiency of routing algorithms. However, disseminating routing information across the network comes with overhead costs. This fact was not even considered by the authors. 
In order to solve the power control problem for nodes with heterogeneous distribution, Kawadia and Kumar [30] have proposed a protocol called CLUSTERPOW. In clustered networks, CLUSTERPOW protocol allows nodes to use a power level which depends on the destination of the packet so as to maximize spatial reuse and hence network capacity. In particular, every node forwards a packet for a destination $d$ using the smallest power level $p$ such that the destination $d$ is reachable, possibly in multiple hops, using only $p$. In Kawadia and Kumar [14], the authors have generalized the solution by [30]. They have discussed the COMPOW, CLUSTERPOW, MINPOW and LOADPOW power control protocols. In this case, the MINPOW attempts to optimize the total energy consumption globally [144, 145]. In order to achieve this goal, they have presented an architecturally clean implementation of the MINPOW in Linux without assuming any physical layer support. Instead, estimates of link costs are performed using control packets at the network layer. In order to maximize network capacity by increasing spatial reuse, a power control adaptive to the traffic load called LOADPOW is introduced. The LOADPOW protocol opportunistically uses a higher transmit power level whenever the network load is low, and lowers the transmit power as the load increases. The authors have given detailed implementations of these protocols in wireless IEEE 802.11 cards, test-bed experimentation using several topology scenarios, and discussed their performance. Although running routing daemons at each power level have shown no loop cycles, such iterative computations coupled with table maintenance can eventually increase route computation delay.

Park and Sivakumar [161, 162] have argued that the use of minimum transmission range and hence MINPOW protocol might not always result in an optimal throughput. Using throughput and throughput per unit energy optimization criteria, they have demonstrated that the optimal transmission power is generally a function of the number of stations, the network size, and the traffic load. As a result, they have defined analytical throughput in terms of spatial reuse, hop count and contention time. They have substantiated their arguments through a comprehensive set of simulation results in both typical and atypical network configurations in terms of number of stations and network density.

Power-Aware Routing in wireless networks that address the inherent conflict between energy efficient communication and the need to achieve desired QoS such as end-to-end communication delay has been thoroughly investigated [137, 163]. Of interest, Chipara et al., [163] have suggested a real-time power-aware routing (RPAR) protocol that dynamically adapts transmission power and route packets based on packet deadlines. The RPAR has salient features including improving the number of packets meeting their deadlines at low energy cost and using an efficient neighbour manager that quickly discovers forward choices (pairs of a neighbour and a transmission power) that meet packet deadlines while introducing low communication and energy overheads. However, it is difficult to acquire an eligible neighbour manager that meets the stringent velocity requirements. Simulations based on MICA2 motes have shown that RPAR reduces miss ratio and energy consumption [117].

Gomez and Campbell [137] have studied the impact of individual variable-range transmission power control on the physical and network connectivity, network capacity and power savings in wireless multi-hop networks. The authors have shown that the average traffic carrying capacity of nodes remains constant even when nodes are added to the network. 
They have also shown that the ratio between the minimum transmission range levels obtained using common-range and a variable-range based routing protocol is approximately two. They have also derived a model that approximates the signalling overhead of a routing protocol as a function of the transmission range and topology dynamics for both route discovery and route maintenance. They have concluded that, routing protocols based on common-range transmission power, limit the capacity available to nodes [164]. In spite of desirable features, the use of variable range may result in medium access unfairness whereby the lower range nodes are suppressed by the higher range nodes.

In, Li et al., [165] a multi-rate power controlled MAC protocol, called MRPC-MAC to enable fairness and a multi-rate power controlled routing, called MRPC-Routing to determine the next hop right before transmitting packets at the MAC layer has been proposed. The MRPC-MAC and Routing use the Effective Transport Capacity as the routing metric so that short links with high bandwidth are preferred and more concurrent transmissions can be enabled. Although spatial reuse and network throughput could significantly be improved, authors have not shown how the protocol performs in case of all short links with low bandwidth.

The problem of joint power control, scheduling and routing in multi-hop wireless networks has received much attention (e.g., [25, 166-170]). The chief objective has been the need to reduce energy consumption of individual nodes and the overall network without severely degrading network throughput (i.e., capacity). In connection with this, [168] has exploited two multi-hop routing protocols. One routing protocol determines packet routes according to a shortest-path criterion and aims to minimize the total transmission power. The other routing protocol selects routes that minimize the cumulative energy consumption within the network (i.e., both links and nodes). The algorithm developed jointly performs link scheduling and power control in order to minimize packet delays and transmission powers. However, wireless sensor nodes (WSNs) are assumed that transmit application information to or receive control information from a central node. This is not feasible for Ad Hoc like WBMNs.

In case where the exact end-to-end traffic matrix is unknown, Kashyap et al., [169] have proposed algorithms that compute a two-phase routing, schedule and power assignment. Their goal is to minimize the total transmission power in the network over all traffic matrices in a given polytope. They have proved the algorithms to be 3-approximations with respect to an optimal algorithm. The drawback is that although the scheme does not require the network to detect changes in the traffic distribution, the limits imposed by the ingress-egress nodes on capacity bounds need to be known across each node in the network. However, overhead-efficient means to do this has not been investigated.

Cruz and Santhanam [166] studied the problem of joint routing, link scheduling and power control to support high data rates for broadband wireless multi-hop networks. Authors have applied the convex optimization problem whereby link scheduling and power control policies are optimized, subject to given constraints (i.e., the minimum average data rate per link and peak transmission power constraints per node). They have found the sensitivity of the minimal total average power with respect to the average data rate for each link. They have noted that shortest path algorithms with link weights set to the link sensitivities can be used to 
guide the search for optimum routing globally. In this case, it can be found out that, optimal allocations do not necessarily route traffic over minimum energy paths. With single channel wireless mesh networks in mind, Gupta [167] designed a joint mechanism that does routing in parallel with the scheduling, by calculating routes over links that fit completely into the current schedule. The power control algorithm for both these schemes is to use the minimum power level needed for communication. None the less, the algorithm does not necessarily produce optimal power values.

In Yuan et al., [20], a joint optimization of multicast routing and power control for wireless mesh networks have been suggested. The aim is to maximize multicast throughput and propose a cross-layer optimization model and solve the optimal throughput problem in an efficient and distributed manner. Authors discussed geometric programming method and game-theoretic approach to solve the transmission power control problem. Their contribution was to strike a balance between the demand of link bandwidth at the network layer and the supply of link capacity at the physical layer. Though multicast ensures reaching multiple receivers in a single transmission, algorithms proposed demanded information exchange by broadcasting messages. Broadcasting introduces network flooding even if it is localized and thus exacerbates the cross-layer optimization problems.

$\mathrm{Xi}$ and Yeh [170] have proposed optimal distributed power control, routing and congestion control in wireless networks. Authors have assumed a multi-commodity flow model in which power and routing variables are chosen to minimize convex link costs reflecting an average queuing delay. Distributed network algorithms performing joint power control and routing on a node-by-node basis have been established. The benefit of their work is that congestion control can seamlessly be incorporated to optimize the user input rates [21].

$\mathrm{Xi}$ and Yeh [171] have devised a spectrum allocation scheme that divides the whole spectrum into multiple sub-channels and activates conflict-free links on each sub-band. They have then proposed a simple distributed and asynchronous algorithm to feasibly activate links on the sub-bands. For the active links on each sub-band, authors developed an optimal power control, traffic routes' and user input rates' algorithms based on the channel states and traffic demands. They have proved that under specific conditions the algorithms asymptotically converge to optimal operating points. However, this convergence performance has been subjected to specified conditions and thus may not hold for general conditions [73].

\subsubsection{Summary}

In summary, joint cross-layer resource optimization involves allocations of transmit power level, MAC layer schedules, links, input rates, routes and delay constraints [170, 171]. The benefits are energy-management, throughput improvement and connectivity preservation. However, many cross-layer optimizations from the physical layer to application layer lacks proper modularity and scalability. Complex models are presented and sub-optimal solutions are obtained [19]. In order to ensure modularity and scalability new DTPC techniques at the link layer need to be researched further.

\section{Conclusions and Remarks}

The DTPC problems for the wireless networks have been extensively addressed in the 
literature. The motivations can be outlined as follows. Firstly, in order to compensate effects of time-varying random wireless channels and thus high SINR. Secondly, in order to minimize energy consumption and thus elongate network lifetime. Thirdly, in order to reduce MAC problems such as exposed terminal nodes (ETNs), hidden terminal nodes (HTNs), collisions and contentions and spectrum sharing fairness and thus maximize spatial reuse (network capacity). Fourthly, in order to improve connectivity attributes such as minimal average node degree and/or fault-tolerant network connectivity and thus energy-efficient routing. Fifthly, in order to minimize congestion and thus optimal end-to-end network throughput and latency. These objectives have been formulated as an optimization problem which tries to strike a balance between minimal use of power at a node and maximal network throughput gain. The attributes of the DTPC optimality are highlighted as follows. The power level should be high enough to avoid interference from the HTPs but no higher so that it will create unnecessary contention or collision among nodes in a shared channel. Furthermore, the power level should be low enough to save energy but no lower so that it will create a disconnected network. Finally, the transmission power adjustment should be fast enough to eliminate queue delays but no faster so that it will create queue singular perturbations among multiple radios. Optimal solutions have been conducted based on a number of conceptual frames: mathematical programming, game theory, dynamic control theory and cross-layering protocol heuristics. Nonetheless there are still a number of open issues for WBMNs.

- In order to address both forward and backward power controlled message exchanges, the Link State Information (LSI) measurement protocols need to take into account bi-directional information exchanges.

- In order to address multiple LSI based optimal DTPC algorithms, it is necessary to describe the LSI by SIR error, aggregate Interference, and connectivity range and link rate deviations. The LSI can then be viewed as state space models so that control theory can then be applied.

- In order to address protocol modularity, scalability and delay a cross multiple layers, the DTPC algorithm should be implemented at the Link-Layer of the protocol stack.

- In order to access the mesh client networks and route the backbone traffic simultaneously, each backbone may be configured with dual or multiple radios and an energy-efficient unification protocol should be developed at the Link-Layer.

- In order to ameliorate singular perturbations between power and packet dynamics at the queues, the DTPC protocol should be developed to manage such system instability.

\section{References}

[1] Akyildiz I.F., Wang X. and Wang W., "Wireless Mesh Networks: a Survey". Computer Networks. Vol. 47, Issue 4. Pp 445-487. 2005.

[2] Haas Z.J., Wireless Ad Hoc Networks: Institute of Electrical and Electronics Engineers (IEEE), 1999.

[3] Akyildiz I.F., Su W., Sankarasubramaniam Y. and Cayirci E., "Wireless Sensor Networks: a survey". Computer Networks. Vol. 38, Issue 4. Pp 393-422. 2002.

[4] Gungor V.C., Natalizio E., Pace P. and Avallone S., "Challenges and Issues in Designing Architectures and Protocols for Wireless Mesh Networks", in Wireless Mesh Networks: architecture and protocols, 
Spinger, 2008.

[5] Akyildiz I.F. and Wang X., Wireless Mesh Networks: John Wiley \& Sons Ltd, 2009.

[6] Sorooshyari, S. and Gajic Z., "Autonomous Dynamic Power Control for Wireless Networks: User-centric and Network centric consideration", IEEE Trans. On Wireless Communications. Vol. 7, Issue 3. Pp 1004-1015. March 2008,

[7] Chiang M., Hande P., Lan T. and Tan C.W., Power Control in Wireless Cellular Networks, Foundation and Trends in Networking, Hanover MA 02339 USA: now Publishers Inc, 2008.

[8] Gunnarsson F., "Power Control in Cellular Radio Systems: Analysis, Design and Estimation", Electrical Engineering, Linkoping Sweden: Linkoping Studies in Science and Technology, 2000, pp. 262.

[9] Koskie S. and Gajic Z., "Signal-To-Interference-Based Power Control for Wireless Networks: a Survey, 1992-2005", Dynamics of continuous, Discrete and Impulsive Systems B: Applications and Algorithms . Vol. 13, Issue 2. Pp. 187-220. 2006.

[10] Koskie S. and Gajic Z., "Optimal SIR-Based Power Control Strategies for Wireless CDMA Networks", International Journal of Information and Systems Sciences. Vol. 1, Issue 1, Pp 1-18. 2007.

[11] Correia L.T.H.A., Macedo D.F., Silva D.A.C., Santos A.L.D., Loureiro A.A.F. and Noguerira J.M.S., "Transmission Power Control in MAC Protocols for Wireless Sensor Networks", Proc. of the 8th ACM/IEEE International Symposium on Modelling, Analysis and Simulation of Wireless and Mobile Systems (MSWiM’05). Montreal, Quebec, Canada, 2005. Pp 282-289.

[12] Khemapech I., Miller A. and Duncan I., "A Survey of Transmission Power Control in Wireless Sensor Networks", Proc. of the 8th Annual Postgraduate symposium on the Convergence of Telecommunications, Networking and Broadcasting (PGNet 2007). 2007. Pp. 15-20.

[13] Pantazis N.A., Vergados D.D., Miridakis N.I. and Vergados D.J., "Power control schemes in wireless sensor networks for homecare e-health applications", ACM International Conference Proceeding Series, Athens, Greece, 2008.

[14] Kawadia V. and Kumar P. R., "Principles and Protocols for Power Control in Ad hoc Networks". IEEE Journal on Selected Areas in Communications. Vol. 23, Issue 5. Pp. 78-88. 2005.

[15] Krunz M. and Muqattash A., "Transmission Power Control in Wireless Ad Hoc Networks: Challenges, Solutions, and Open Issues". IEEE Network. Vol. 18, Issue5. Pp. 8-14. 2004.

[16] Monks J.P., "Transmission power control for enhancing the performance of wireless packet data networks". Faculty of Engineering, Urbana-Champaign: University of Illinois at Urbana-Champaign, 2001.

[17] Narayanaswamy S., Kawadia V., R.S. Screenivas R.S. and Kumar P.R., "Power Control in Ad Hoc Networks: Theory, Architecture, Algorithm and Implementation of the COMPOW Protocol", Proceedings of the European Wireless Conference, Florence, Italy. Pp. 156-162. 2002.

[18] Capone A. and Martignon F., "Power-controlled Directional Medium Access Control for Wireless Mesh Networks", in Wireless System and Network Architecture, Berlin: Springer Berlin, 2006, pp. 34-46.

[19] Georgiadis L., Neely M.J. and Tassiulas L., Resource Allocation and Cross-Layer Control in Wireless Networks, Foundations and Trends in Networking, USA: now Publishers Inc., 2006.

[20] Yuan J., Li Z., Yu W. and Li B., "A cross-layer Optimization Framework for Multi-hop multi-cast in Wireless Mesh Networks". IEEE Journal Selected Areas in Communications. Vol. 24, Issue 11. 2006.

[21] Chiang M., "Balancing Transport and Physical Layers in Wireless Multi-hop Networks: Jointly Optimal Congestion Control and Power Control". IEEE Journal on Selected Areas in Communications. 
Vol. 23, Issue 1. 2005.

[22] Chiang M., Tan C.W., Palmar D.P., O’Neill D. and Julian D., "Power Control by Geometric Programming". IEEE Trans. Wirel. Communications. Vol. 6, Issue 7, Pp. 2640-2651. 2007.

[23] Montemanni R. and Gambardella L.M., "Minimum Power Symmetric Connectivity problem in wireless networks: a new approach", in Mobile and Wireless Communication Networks: Springer, 2004, pp. 496-508.

[24] Fischione C., and Butussi M., "Power and Rate Control Outage Based in CDMA Wireless Networks under MAI and Heterogeneous Traffic Sources", Proc. of IEEE International Conference on Communications 2007 (IEEE ICC 07). Glasgow, UK, 2007.

[25] Shi Y., and Hou Y.T., "Optimal Power Control for Multi-hop software Defined Radio Networks", Technical Report, Virginia: Bradley Department of ECE, Virginia Tech., 2006.

[26] Ares B.Z., Park P.G., Fischione C., Speranzon A. and Johansson K.H., "On Power Control for Wireless Sensor Networks: System Model, Middleware Component and Experimental Evaluation", Proc. of IFAC European Control Conference, Kos, Greece, 2007.

[27] Arora A. and Krunz M., "Power-Controlled medium access for Ad Hoc networks with directional antennas". Ad Hoc Networks. Vol. 5. Pp. 145-161. 2007.

[28] Dongsheng L., Yong X. and Meilin S., "A Multi-Channel Based Power Control Protocol for Mobile Ad Hoc Networks", Proc. International Conference on Wireless Networks (ICWN’2006), Las Vegas, USA, 2006, pp. 237-243.

[29] Gomez J., Campbell A.T., Naghshineh M. and Bisdikian C., "PARO: Supporting Dynamic Power Controlled Routing in Wireless Ad Hoc Networks". ACM/Kluwer, Journal Wireless Networks. Vol. 9, Issue 2003. Pp. 443-460. 2003.

[30] Kawadia V. and Kumar P.R., "Power control and clustering in Ad Hoc networks", Proc. IEEE INFOCOM 2003 Conference, San Fransisco, California, USA, 2003, pp. 459-469.

[31] Muqattash A. and Krunz M., "POWMAC: A Single-Channel Power-Control Protocol for Throughput Enhancement in Wireless Ad Hoc Networks". IEEE Journal, Selected Areas in Communications, (JSAC)-Special Issue on Advances in Military Communications. Vol. 23, Issue 5. Pp. 1067-1084. 2005.

[32] Tadonki C. and Rolim J., "Integer Programming Heuristic for the Dual Power Setting Problem in Wireless Sensors Networks". Sensors Networks. Vol. 1, Issue2. 2008.

[33] Montemanni R., Gambardella L.M. and Das A.K., "The Minimum Power Broadcast problem in Wireless Networks: a Simulated Annealing approach", Proc. Of the IEEE Wireless Communications and Networking Conference (WCNC’2005), New Orleans, USA, 2005.

[34] Altman E., Avrachenkov K., Bonneau N., Debbah M., El-Azouzi R. and Menasche D., "Constrained Stochastic Games in Wireless Networks". Technical Report, 2007: www.cs.umass.edu/ sadoc/mdp, 2007.

[35] Srivastava V., Neel J., MacKenzie A.B., Menon R., DaSilva L.A, Hicks J.E., Reed J.H. and Gilles R.P., "Using Game Theory to Analyze Wireless Ad Hoc Networks". IEEE Communications Surveys and Tutorials 4th Quarter 2005, 2005.

[36] Wang Y. and Li X.-Y.,"Minimum Power Assignment in Wireless Ad Hoc Networks with Spanner Property". Kluwer Academic Publishers, Netherlands, Pp. 1-15. 2005.

[37] Xing Y. and Chandramouli R., "Stochastic Learning Solution for Distributed Discrete Power Control Game in Wireless Data Networks", Proceedings of IEEE International Conf. on Communications (ICC), Paris, 2004. 
[38] Shoarinejad K., Speyer J.L. and Pottie G.J., "A distributed scheme for integrated predictive dynamic channel and power allocation in cellular radio networks", Proc. Global Telecommunication (GLOBECOM 2001) Conference, San Antonio, 2001, pp. 3623-3627.

[39] Leung, K.K., "A Kalman-filter method for power control in broadband wireless networks", Proc. IEEE INFOCOM 1999 Conference, New York, 1999, pp. 948-956.

[40] Leung, K.K., "Power Control by interference predictions for wireless packet networks", IEEE Trans. Wireless Communications. Vol. 1, Issue 2, Pp. 256-265. 2002.

[41] Leung, K.K., "Power Control by Kalman Filter with Error Margin for Wireless IP Networks". US patent, 2003.

[42] Subramanian A. and Sayed A.H., "Joint Rate and Power Control Algorithms for Wireless Networks". IEEE Trans. On Signal Processing. Vol. 53, Issue 11. 2005.

[43] Altman E., Avrachenkov K., Millen G. and Prabhu B., "Discrete Power Control: Cooperative and Non-Cooperative Optimization", Proc. IEEE INFOCOM 2007 Conference, Alaska, USA, 2007.

[44] Fischione C., Butussi M., Johansson K.H. and D’Angelo M., "Power and Rate Control with Outage Constraints in CDMA Wireless Networks". IEEE Transactions on Communications. 2009.

[45] Tang J., Xue G., Chaudler C. and Zhang W., "Link Scheduling with power control for throughput enhancement in multi-hop wireless networks". IEEE Transaction Vehicular Technology. Vol. 55, Issue 3. Pp. 733-742. 2006,

[46] Ramamurthi V., Reaz A., Dixit S. and Mukherjee B., "Link Scheduling and Power Control in Wireless Mesh Networks with Directional Antennas", Proc. IEEE ICC 2008 conference, Beijing, China, 2008, pp. 4835-4839.

[47] Montemanni R. and Gambardella L.M.,"Exact algorithms for the minimum power symmetric connectivity problem in wireless networks", Computers and Operations Research. Vol. 32, Issue11, Pp. 2891-2904. 2005.

[48] Montemanni R., Gambardella L.M. and Das A.K., "Models and exact algorithms for the min-power symmetric connectivity problem: an overview", in Handbook on Theoretical and Algorithmic Aspects of Sensor, Ad Hoc Wireless, and Peer-to-Peer Networks: Auerbach Publications, 2006, pp. 133-146.

[49] Wieselthier J., Nguyen G. and Ephremides A., "On the construction of energy-efficient broadcast and multi-cast trees in wireless networks", Proc. IEEE INFOCOM 2000 Conference. Israel, 2000, pp. 585-594.

[50] Tadonki C. and Rolim J., "Integer programming heuristic for the dual power management problem in sensor networks", Proc. 2nd International Workshop on Managing Ubiquitous Communication and Services (MUCS'2004). Dublin, 2004.

[51] Carello, A.C., "Scheduling Optimization in Wireless Mesh Networks with Power Control and Rate Adaptation", Proc. IEEE SECON 2006 conference. Reston, USA, 2006.

[52] Vasudevan S., Zhang C., Goeckel D. and Towsley D., "Optimal power allocation in wireless networks with transmitter-receiver power tradeoffs", Proc. IEEE 25th International Conference on Computer Communications (INFOCOM’06). Barcelona, 2006, pp. 1-11.

[53] Shu T., Krunz M. and Vrudhula S., "Joint optimization of transmit power-time and bit energy efficiency in CDMA wireless sensor networks". IEEE Transactions on Communications. Vol. 5, Issue 11, Pp. 3109-3118. 2006.

[54] Broustis I., Eriksson J., Krishnamurthy S.V. and Faloutsos M., "Implications of Power Control in Wireless Networks: A Quantitative study", Proc. Passive and Active Measurements Conference 
(PAM’07). Louvain-la-neuve, Belgium, 2007.

[55] Koskie, S., "Contributions to Dynamic Nash Games and Applications to Power Control for Wireless Networks": Rutgers University, 2003.

[56] Koskie S. and Zapf J., "Acceleration of Static Nash Power Control Algorithm using Newton Iterations". Dynamics of Continuous, Discrete and Impulse Systems B: Applications and Algorithms. Vol. 12. Pp. 685-690. 2005.

[57] Goodman D.J. and Mandayam N.B., "Network Assisted power control for wireless data". Mobile Networks and Applications. Vol. 6, Issue 5. Pp. 409-418. 2001.

[58] Huang J., Berry R. and Honig M.L., "Performance of Distributed Utility-Based Power Control for Wireless Ad Hoc Networks", Proc. Military Communication Conference (MILCOM'2005), Atlantic City, New Jersey, 2005.

[59] Huang J., Berry R.A. and Honig M.L., "A Game Theoretic Analysis of Distributed Power Control for Spread Spectrum Ad Hoc Networks", Proc. IEEE International Symposium Information Theory (ISIT 2005) Conference. Adelaide, Australia, 2005.

[60] Jean C.A.S. and Jabbari B., "Game-Theoretic Delay-Sensitive Multi-rate Power Control for CDMA Wireless Networks with Variable Path Loss ". Proc. IEEE Wireless Communications and Networking Conference (WCNC). Las Vegas, Nevada, 2006.

[61] Meshkati F., Chiang M., Poor H.V. and Schwartz S.C., "A Game-Theoretic Approach to Energy-Efficient Power Control in Multi-carrier CDMA systems". IEEE Journal Selected Areas in Communications, Vol. 24, Issue 6. Pp. 1115-1129. 2006.

[62] Famolari D., Mandayam N.B., Goodman D.J. and Shah V., "A new framework for power control in wireless data networks: games, utility and pricing", in Wireless multimedia network technologies, Kluwer Academic Publishers, 1999, pp. 289-310.

[63] Liang C. and Dandekar K.R.,"Power Management in MIMO ad hoc networks: a game theoretic approach". IEEE Transaction on Wireless Communication. Vol. 6, Issue 4. Pp. 1164-1170. 2007.

[64] Thomas R.W., Komali R.S, MacKenzie A.B. and DaSilva L.A., "Joint Power and Channel Minimization in Topology Control: A cognitive Network Approach", Proc. IEEE International Conference on Communication (ICC’2007), Glasgow, Scotland, 2007, pp. 6538-6542.

[65] Closas, P., Pages-Zamora A. and Fernadez-Rubio J.A., "A game theoretical algorithm for joint power and topology control in distributed WSN", Proc. IEEE International Conference on Acoustics, Speech and Signal Processing (ICASSP’09). Taipei, Taiwan, 2009, pp. 2765-2768.

[66] Huang J., Han Z., Chiang M. and Poor H.V., "Distributed Power Control and Relay Selection for Cooperative Transmission Using Auction Theory". IEEE Journal Selected Areas in Communications. Vol. 26, Issue 7. Pp. 1226-1237. 2008.

[67] Jindal N., Weber S. and Andrews J.G., "Fractional Power Control for Decentralized Wireless Networks". IEEE Transactions on Wireless Communications, Vol. 7, Issue 12. Pp. 5482-5492. 2008.

[68] Neely M.J., Modiano E. and Rohrs C.E, "Dynamic Power Allocation and Routing for Time-Varying Wireless Networks". IEEE Journal on Selected Areas of Communication. Vol. 23, Issue 1. Pp. 89-103. 2005.

[69] Olama M.M., Djouadi S.M. and Charalambous C.D., "Stochastic power control for time-varying long-term fading wireless". EURASIP Journal on Applied Signal Processing. Vol. 2006, Pp. 1-13. 2006.

[70] Holliday T., Goldsmith A., Glynn P. and Bambos N., "Distributed Power and Admission control for 
time varying wireless networks", Proc. IEEE International Symposium on Information Theory (ISIT’04), Chicago, Illinois, 2004.

[71] Lin S., Zhang J., Zhou G., Gu L., He T. and Stankovic J.A., "ATPC: Adaptive Transmission Power Control for Wireless Sensor Networks", Proc. 4th ACM Conf. Embedded Networked Sensor (SenSys’06). Boulder, Colorado, USA: ACM, 2006.

[72] Foschini G.J. and Miljanic Z., "A simple distributed autonomous power control algorithm and its convergence". IEEE Trans. On Vehicular Technology. Vol. 42,Issue 4. Pp. 641-647. 1993.

[73] Yates, R.D., "A framework for uplink power control in cellular radio systems". IEEE Journal on Selected Areas in Communications. Vol. 13, Issue 7. Pp. 1341-1348. 1995.

[74] Kim, D., "Rate regulated power control for supporting flexible transmission in future CDMA mobile networks". IEEE Journal on Selected Areas Communication. Vol. 17, Issue 5. Pp. 968-977. 1999.

[75] Olama M.M., Shajaat S.M., Djouadi S.M. and Charalambous D., "Stochastic power control for time-varying short term flat fading wireless channels", Proc. 16th IFAC World Congress. Prague, Czech Republic, 2005.

[76] Su H.-J. and Geraniotis E., "Adaptive closed-loop control with quantized feedback and loop filtering". IEEE Trans. Wireless Communications. Vol. 1, Issue 1. Pp 76-86. 2002.

[77] Agarwal S., Katz R.H., Krishnamurthy S.V. and Dao S.K., "Distributed power control in Ad-Hoc wireless networks", Proc. IEEE 12th International Symposium on Personal, Indoor and Mobile Radio Communications. 2002.

[78] Hande P., Rangan S., Chiang M. and Wu X., "Distributed uplink power control for optimal SIR assignment in cellular data networks". IEEE/ACM Transactions on Networking. Vol. 16, Issue 6. Pp 1430-1443. 2008.

[79] Aldajani M.A. and Sayed A.H., "Adaptive Predictive Power Control for the Uplink Channel in DS-CDMA cellular systems". IEEE Trans. Vehicular Technology. Vol. 52, Issue 6. Pp 1447-1463. 2003.

[80] Shoarinejad K., Speyer J.L. and Pottie G.J., "An Integrated Predictive Power Control and dynamic channel assignment in mobile radio systems". IEEE Transactions on Wireless Communications. Vol. 2, Issue 5. Pp 976-988. 2003.

[81] Shoarinejad, K., "On Stochastic Decentralised Systems in Communications and Controls", Electrical Engineering Department: University of California Los Angeles, 2001.

[82] Leung K.K., Driesson P.F. and Chaula K., "Link Adaptation and Power Control for Streaming Services in EGPRS wireless networks". IEEE Journal Selected Areas in Communication. Vol. 19. Pp 2029-2039. 2001.

[83] Chiang J.C., Leung K.K. and Qiu X., "Power control for packet voice service with application to EDGE wireless system". International Journal of Wireless Information Networks. Vol. 11. Pp 29-39. 2004.

[84] Cheng H.T., Jiang H. and Zhuang W., "Distributed medium access control for wireless mesh networks". Journal of Wireless Communications and mobile computing. Vol. 6. Pp 845-864. 2006.

[85] Jung E.-S. and Vaidya N.H., "A power control MAC protocol for ad-hoc networks", Proc. ACM 8th Annual International Conf. on Mobile Computing and Networking (MOBICOM’02). Atlanta, Ga, USA, 2002, pp. 36-47.

[86] Chen H.-H., Fan Z. and Li J., "Autonomous power control MAC protocol for mobile ad hoc networks". EURASIP Journal on Wireless Communications and networking. Vol. 2006. Pp. 1-10. 2006.

[87] Sheth A. and Han R., "SHUSH: Reactive transmission power for wireless MAC protocols", Proc. IEEE 
1st International Conference on the Wireless Internet (WICON’05). Budapest, Hungary, 2005, pp. 18-25.

[88] Muqattash A. and Krunz M., "Power controlled dual channel (PCDC) medium access protocol for wireless ad hoc networks", Proc. IEEE INFOCOM 2003 Conference. San Francisco, 2003.

[89] Muqattash A. and Krunz M., "A distributed transmission power control protocol for mobile ad hoc networks". IEEE Transactions on Mobile Computing. Vol. 3, Issue 2. Pp 113-128. 2004.

[90] Siam M. and Krunz M., "Throughput-Oriented power control in MIMO-based ad hoc networks", Proc. IEEE ICC 2007 Conference: Wireless Ad Hoc and Sensor Symposium, Glasgow, Scotland, 2007.

[91] Ding P., Holliday J. and Celik A., "Demac: an adaptive power control MAC protocol for Ad Hoc networks", Proc. IEEE 16th International Symposium Personal, Indoor Mobile Radio Communication (PIMRC’05), Berlin, Germany, 2005.

[92] Jia L., Liu X., Noubir G. and Rajaraman R., "Transmission power control for Ad Hoc wireless networks: throughput, energy and fairness", Proc. IEEE Wireless Communications and Networks Conference (WCNC’05), New Orleans, LA, USA, Pp 619-625. 2005.

[93] Li P., Gang X. and Fang Y., "An Adaptive power controlled MAC protocol for wireless Ad Hoc networks". IEEE Transactions on Wireless Communication. Vol. 8, Issue 1. Pp 226-233. 2009.

[94] Qiao D., Choi S. and Shin K.G., "Interference analysis and transmit power control in IEEE 802.11a/h wireless LANs". IEEE/ACM Transaction Network. Vol. 15,Issue 5. Pp 1007-1020. 2007.

[95] Ho W.H. and Liew S.C., "Distributed Adaptive Power Control in IEEE 802.11 Wireless Networks", Proc. IEEE International Conference on Mobile Ad-Hoc and Sensor System (MASS’06). Vancouver, Canada, 2006.

[96] Ho W.H. and Liew S.C., "Impact of power control on performance of IEEE 802.11 Networks". IEEE Transaction on Mobile computing. Vol. 6, Issue 11. Pp 1245-1258. 2007.

[97] Ho W.H. and Liew S.C., "Achieving Scalable Capacity in Wireless Networks with Adaptive Power Control", Proc. IEEE 5th International Workshop on Wireless Local Networks (WLN). 2005, pp. 720-728.

[98] Fu L., Liew S.C. and Huang J., "Fast Algorithms for joint power control and scheduling in wireless networks". To appear in IEEE Transactions on Wireless Communication, 2010.

[99] Polastre J., Hill J. and Culler D., "Versatile low power medium access for wireless sensor networks", Proc. 2nd International Conf. on Embedded networked sensor systems. 2004, pp. 95-107.

[100] Correia L.T.H.A., Macedo D.F., Silva D.A.C., Santos A. L. D., Loureiro A.A.A., and Noguerira J.M.S., "Challenges and Experiences in the Design of Transmission Power Control Protocols for Wireless Sensor Networks", DCC/UFMG-Technical Report RT. DCC. 02/2006: Department of Computer Science, Federal University of Minas Gerais, Brazil, 2006.

[101] Jeong J., Culler D. and Oh J.-H., "Empirical Analysis of Transmission Power Control Algorithms for Wireless Sensor Networks", Proc. 4th International Conference on Networked Sensing Systems (INSS’07). Braunschweig, Germany: Piscataway, 2007, pp. 27-34.

[102] Hasan A., Yang K. and Andrews J.G., "Clustered CDMA AD HOC networks without closed-loop power control", Proc. IEEE MILCOM 2003 Conference, Boston, 2003.

[103] Lai W.K. and Li C., "PCIA: A Power control interference avoidance scheme for ad hoc networks", Proc. International Conference on Wireless Networks (ICWN’06). Las Vegas, USA, 2006, pp. 186-192.

[104] Wu S.-L., Tseng Y.-C, Lin C.-Y. and Shen J.-P., "A multi-channel MAC protocol with power control for multi-hop mobile ad hoc networks". The Computer Journal. Vol. 45, Issue 1. Pp 101-110. 2002. 
[105] Kim Y. Shin H. and Cha H., "Y-MAC: An Energy-Efficient Multi-Channel MAC protocol for dense wireless sensor networks", Proc. of 7th International Conference on Information Processing in Sensor Networks, St. Louis, Missouri, USA, 2008, pp. 53-63.

[106] Tang Z., Hu Q. and Yu G., "Power control strategies for multi-channel cognitive wireless networks with opportunistic interference cancellation". Journal of Electronics (China) .Vol. 25, Issue 2. Pp. 268-273. 2008.

[107] Zhang Y. and Qu S., "Joint power control and channel assignment algorithms for wireless ad hoc networks", in Ubiquitous Intelligence and Computing, Berlin: Springer Berlin / Heidelberg, 2006, pp. 270-279.

[108] Olwal T., Wyk B.J.V., Djouani K., Hamam Y., Siarry P. and Ntlatlapa N., "A Multi-State Based Power Control for Multi-Radio Multi-Channel Wireless Mesh Networks". International Journal of Computer Science (WASET). Vol. 4, Issue 1. Pp. 53-61. 2009.

[109] Arora A., Krunz M. and Muqattash A., "Directional Medium Access Protocol with Power Control for Wireless Ad Hoc Networks", Proc. IEEE Global Telecommunication Conference (Globecom’04), Dallas, USA, 2004, pp. 2797-2801.

[110] Choudhary R.R., Yang X., Ramanathan R. and Vaidya N.H., "Using directional antennas for media access control in ad hoc networks", Proc. ACM MobiCom (MobiCom'02) Conference, Atlanta, Georgia, USA, 2002, pp. 59-70.

[111] Capone A., Martignon F. and Fratta L., "Directional MAC and Routing Schemes for Power Controlled Wireless Mesh Networks with adaptive Antennas". Ad Hoc Networks Journal. 2008.

[112] Alawieh B., Assi C. and Mouftah H., "Power-aware ad hoc networks with directional antennas: Models and Analysis". Ad Hoc Networks. Vol. 7, Issue 3. Pp 486-499. 2009.

[113] Fahmy N.S., Todd T.D. and Kezys V., "Distributed power control for ad hoc networks with smart antennas", Proc. IEEE Vehicular Technology Conference (VTC). Vancouver, BC, Canada, 2002, pp. 2141-2144.

[114] Lai W.K., Tsai H.-S., Li C., and Shieh C.-S., "A power efficient solution to the large interference range problem in ad hoc networks", Journal of Wireless Communications and Mobile Computing .Vol. 9, Issue 9. Pp. 1264-1273. 2009.

[115] Alawieh, B., Assi C., and Mouftah H., "Investigating the performance of power-aware IEEE 802.11 in Multi-hop wireless networks". IEEE Transactions on Vehicular Technology. Vol. 58,Issue 1. Pp. 287-300. 2009.

[116] Ha R.H., Ho P.-H. and Shen X.S., "Optimal sleep scheduling with transmission range assignment in application-specific wireless sensor networks". To appear in International Journal Sensor Networks. Pp. 1-17. 2010.

[117] Zheng R., Hou J.C. and Li N., "Power management and power control in wireless networks", in Ad Hoc and Sensor Networks: Nova Science Publishers, 2006, pp. 1-25.

[118] Jamieson K., Chen B., Balakrishnan H. and Morris R., "Span: Topology Maintenance for Energy Efficiency in Ad Hoc Wireless Networks", Proc. ACM MobiCom 2001 conference. Rome, Italy, 2001.

[119] Xu Y., Bien S., Mori Y., Heidemann J. and Estrin D., "Topology Control Protocols to Conserve Energy in Wireless Ad Hoc Networks", Technical Report 6, Los Angeles: University of Los Angeles, 2008.

[120] Hsu H.-Y. and Hurson A.R., "PEAN: A Probabilistic Energy Aware Neighbour Monitoring Protocol for Mobile Ad Hoc Networks", Proc. IEEE 21st International Conference Advanced Information Networking and Applications (AINAW’07). Niagara Falls, Ontario, 2007, pp. 201-206. 
[121] Feeney, L.M., "An Asynchronous Power Save Protocol for Wireless Ad Hoc Networks", SICS Technical Report: T2002:9, SICS 2002, pp. 1-31.

[122] Feeney L.M., Rohner C. and Ahlgren B., "The impact of wakeup schedule distribution in asynchronous power save protocols on the performance of multi-hop wireless networks", Proc. IEEE Wireless Communication Networks Conference (WCNC’07). Hong Kong, 2007.

[123] Dhiman G. and Rosing T.S., "Dynamic power management using machine learning", Proc. International Conference on Computer-Aided Design (ICCAD’06). San Jose, CA, 2006.

[124] Klues K., Xing G. and Lu C., "A Unified architecture for Flexible Radio Power Management in Wireless Sensor Networks", Technical Report: Washington University, School of Engineering, 2006.

[125] Klues K., Xing G. and Lu C., "Link layer support for unified radio power management in wireless sensor networks", Proc. ACM 6th International Conference on Information Processing in Sensor Networks (IPSN’07). Massachusetts, USA, 2007, pp. 460-469.

[126] Xing G., Sha M., Hackmann G., Klues K., Chipara O. and Lu C., "Towards unified radio power management for wireless sensor networks". Wireless Communications and Mobile Computing. 2008.

[127] Xing G., Lu C., Zhang Y. and Huang Q., "Minimum Power Configuration for Wireless Communication in Sensor Networks". To appear ACM Journal. pp. 1-29. 2010.

[128] Zheng R. and Kravets R., "On-demand power management for ad hoc network", Proc. IEEE INFOCOM 2003 Conference, San Franscisco, California, 2003.

[129] Li Z. and Li B., "Probabilistic Power Management for Wireless Ad Hoc Networks". ACM/Kluwer Mobile Networks and Applications (MONET). Vol. 10, Issue 5. Pp 771-782. 2005.

[130] Zhang J., Zhou G., Huang C., Son S.H., and Stankovic J.A., "TMMAC: An Energy Efficient Multi-Channel MAC Protocol for Ad Hoc Networks", ACM SIGMOBILE Mobile Computing and Communications Review (ACM MC2R), Special Issue on Localization Techniques and Algorithms, 2007.

[131] Wang J., Fang Y. and Wu D., "A Power-Saving Multi-Radio Multi-Channel MAC protocol for wireless local area networks", Proc. IEEE INFOCOM 2006 conference, Barcelona, Spain, 2006, pp. 1-13.

[132] Park H., Jee J. and Park C., "Power management of multi-radio mobile nodes using HSDPA interface sensitive APs", Proc. IEEE 11th International Conference Advanced Communication Technology (ICACT’09). Phoenix Park, South Korea, 2009, pp. 507-511.

[133] Miller M. and Vaidya N., "Improving Power Save Protocols Using Carrier Sensing and Busy-Tones for Dynamic Advertisement Window", Technical Report: Technical Report, 2004.

[134] Qiao D. and Shin K.G., "Smart Power-Saving Mode for IEEE 802.11 Wireless LANs", Proc. IEEE INFOCOM 2005 Conference, Miami, 2005.

[135] Bhatia R., Kashyap A. and Li L., "The Power balancing problem in energy constrained multi-hop wireless networks", Proc. IEEE INFOCOM 2007 Conference, Anchorage, Alaska, USA, pp. 553-562.

[136] Ramanathan R. and Hain R.R., "Topology Control of Multi-hop Wireless Using Transmit Power Adjustment", Proc. IEEE INFOCOM 2000 Conference, Tel-Aviv, Israel, 2000, pp. 404-413.

[137] Gomez J. and Campbell A.T., "A case for Variable-Range transmission power control in wireless multi-hop networks", Proc. IEEE International Conference on Computer Communications (INFOCOM'04), Hong Kong, 2004.

[138] Wattenhofer R., Li L., Bahl P. and Wang Y.-M., "Distributed Topology Control for Power Efficient Operation in Multi-hop Wireless Ad Hoc Networks", Proc. IEEE INFOCOM 2001 Conference, Achorage, Alaska, 2001. 
[139] Khan M., Pandurangan G. and Kumar V.S.A., "Energy-Efficient distributed constructions of minimum spanning tree for wireless ad hoc networks", Technical Report 06-019: Department of Computer Science, Purdue University, 2006.

[140] Aron F.O., Olwal T.O., Kurien A. and Odhiambo M.O., "A Distributed Topology Control Algorithm to Conserve Energy in Heterogeneous Wireless Mesh Networks". Proceedings of World Academy of Science, Engineering and Technology. Vol. 30. Pp 530-536. 2008.

[141] Hajiaghayi M., Immorlica N. and Mirrokni V.S., "Power Optimization in Fault-tolarant Topology Control Algorithms for Wireless Multi-hop Networks", Proc. MobiCom’03 Conference. San Diego, California, USA, 2003, pp. 300-312.

[142] Li D., Du H., Liu L. and Huang S.C.-H., "Joint Topology Control and Power Conservation for Wireless Sensor Networks Using Transmit Power Adjustment", in Computing and Combinatorics, Berlin/Heidelberg: SpringerLink, 2008, pp. 541-550.

[143] Tan H.X. and Seah W.K.G., "Dynamic Topology Control to Reduce Interference in MANETs", Institute for Infocomm Research Technical Report: Institute for Infocomm Research Technical Report:NET-2004-AHN-General-0027, 2004.

[144] Calinescu G., Kapoor S., Olshevsky A. and Zelikovsky A., "Summary of Network Lifetime and Power Assignment in ad hoc Wireless networks", 2005.

[145] Calinescu G., Kapoor S., A. Olshevsky A. and Zelikovsky A., "Network Lifetime and Power Assignment in ad hoc Wireless networks", Proc. 11th Annual European Symp. Algorithms (ESA 2003), Berlin: Springer-Verlag, 2003, pp. 114-126.

[146] Choi Y., Khan M., Kumar V.S.A. and Pandurangan G., "Energy-Optimal Distributed Algorithms for Minimum Spanning Trees". IEEE Journal on Selected Areas in Communication, Issue on Stochastic Geometry and Random Graphs in Wireless Networks. Vol. 27, Issue 7. 2009.

[147] Gerharz M., Waal C.D., Frank M. and Martini P., "Influence of Transmission Power Control on the Transport capacity of Wireless Multi-hop Networks", Proc. 75th IEEE International Symposium on Personal, Indoor and Mobile Radio Communication (PIMRC'04), Barcelona, Spain, 2004.

[148] Gerharz M., Waal C.D., Martini P. and James P., "A Cooperative Nearest Neighbours Topology Control Algorithm for Wireless Ad Hoc Networks", Proc. 12th International Conference on Computer Communications and Networks (ICCCN’03) Dallas, TX, USA, 2003, pp. 412-417.

[149] Li N. and Hou J.C., "Localised topology control algorithms for heterogeneous wireless networks", IEEE/ACM Transactions on Networking (TON). Vol. 13, Issue 6. Pp 1313-1324. 2005.

[150] Aron F., Olwal T.O., Kurien A., and Odhiambo M.O., "Network Preservation through a Topology Control Algorithm for Wireless Mesh Networks", Proc. International Conference on Modelling and Simulation (IASTED AfricaMs 2008), Gaborone, Botswana, 2008.

[151] Aron F.O., Olwal T.O., Kurien A. and Odhiambo M.O, "Energy Efficient Topology Control for Wireless Mesh Networks", Proc. IEEE Region 8, International Conference on Wireless Communication and Mobile Computing (IWCMC’2008). Crete, Greece, 2008.

[152] Li X.-Y., Song W.-Z. and Wang Y., "Efficient Topology Control for Ad Hoc Wireless Networks with Non-Uniform Transmission Ranges", 2003.

[153] Yao, A.C.-C., "On constructing minimum spanning trees in k-dimensional spaces and related problems". SIAM Journal of Computing. Vol. 11. Pp 721-736. 1982.

[154] Wang K., Chiasserini C.F., Proakis J.G. and Rao R.R.," Joint scheduling and power control supporting multicasting in wireless Ad Hoc networks". Ad Hoc Networks. Vol. 4. Pp 532-546. 2006. 
[155] Muqattash A., Krunz M. and Lee S.-J., "A Perspective on the design of power control for mobile ad hoc networks",in Mobile Wireless and Sensor Networks: Technology, Applications and Future Directions: John Wiley \& Sons, Inc., 2006, pp. 73-104.

[156] Muqattash A., Krunz M. and Shu T., "On the Performance of Joint rate/power control with adaptive modulation in Wireless CDMA networks", Proc. IEEE INFOCOM 2006 Conference, Barcelona, Spain, 2006.

[157] Li X., Cao F. and Wu D., "QoS-driven power allocation for Multi-channel communication under delayed channel side information", Proc. IEEE Consumer Communications \& Networking (CCNC’09) Conference. Las Vegas, Nevada, USA, 2009.

[158] Li X. and Wu D., "Power control and Dynamic Channel Allocation for Delay Sensitive Applications in Wireless Networks", Proc. IEEE Global Telecommunication Conference (Globecom 2006), San Francisco, CA, USA, 2006.

[159] Chen C.-C. and Lee D.-S., "A Joint Design of Distributed QoS Scheduling and Power Control for Wireless Networks", Proc. IEEE INFOCOM 2006 Conference, Barcelona, Spain, 2006.

[160] Bergamo P., Giovanardi A., Travasoni A., Maniezzo D., Mazzini G. and Zorzi M.," Distributed power control for energy efficient routing in ad hoc networks". Wireless Networks. Vol. 10, Issue 1, 2004.

[161] Park S.-J. and Sivakumar R., "Load-Sensitive Transmission Power Control in Wireless Ad Hoc Network", Proc. IEEE Global Telecommunication Conference (Globecom’02), Taiwan, 2002.

[162] Park S.-J. and Sivakumar R., "Quantitative analysis of transmission power control in wireless ad hoc networks", Proc. IEEE International Conference on Parallel Processing, Vancouver, Canada, 2003.

[163] Chipara O., He Z., Xing G., Chen Q., Wang X., Lu C., Stankovic J. and Abdelzaher T., "Real-time Power-Aware Routing in Sensor Networks", Proc. IEEE 14th International Workshop on Quality of Service (IWQoS’06), Yale University, New Haven, CT, USA, 2006, pp. 83-92.

[164] Savas O., Alanyali M. and Yener B., "Joint route and power assignment in asynchronous multi-hop wireless networks", Proc. MedHocNet 2004 Conference. Bodrum, Turkey, 2004.

[165] Li P., Shen Q., Fang Y. and Zhang H., "Power Controlled Network Protocols for Multi-rate Ad Hoc Networks". IEEE Transaction on Wireless Communication. Vol. 8, Issue 4. Pp 2142-2149. 2009.

[166] Cruz R.L. and Santhanam A.V., "Optimal Routing, Link scheduling and Power Control in Multi-hop wireless networks", Proc. IEEE INFOCOM 2003 Conference, San Francisco, California, 2003.

[167] Gupta, D.K., "Joint scheduling, routing and power control for single-channel wireless mesh networks", Computer Engineering: North Carolina State University, 2006.

[168] Hengstler, S., "Performance of Joint routing, scheduling and power control in energy-constrained wireless sensor networks", Technical Report: Electrical Engineering: Technical Report EE360, 2007, pp. 1-5.

[169] Kashyap A., Senguta S., Bhatia R. and Kodialam M., "Two-phase routing, scheduling and power control for wireless mesh networks with variable traffic", Proc. ACM SIGMETRICS Conference, San Diego, California, USA, 2007, pp. 85-96.

[170] Xi Y. and Yeh E.M., "Optimal Distributed Power Control, routing and congestion control in wireless networks", Technical Report: Department of Electrical Engineering, Yale University, 2006.

[171] Xi Y. and Yeh E.M., "Distributed algorithms for spectrum allocation, power control, routing and congestion control in wireless networks", Proc. ACM 8th International Symposium on Mobile Ad Hoc Networking and Computing, Montreal, Quebec, Canada, 2007, pp. 180-189. 